chapter1-v2

\title{
Gravity beyond general relativity
}

\author{
Kazuya Koyama \\ Institute of Cosmology \& Gravitation, University of Portsmouth, Dennis Sciama Building, \\ Portsmouth, PO1 3FX, United Kingdom \\ Kazuya.Koyama@port.ac.uk \\ Received Day Month Year \\ Revised Day Month Year
}

\begin{abstract}
We introduce the standard model of cosmology based on general relativity and discuss its successes and problems. We then discuss motivations to consider gravitational theories beyond general relativity and summarise observational and theoretical constraints that these theories need to satisfy. A special focus is laid on screening mechanisms, which hide deviations from general relativity in the Solar System and enable large modifications to general relativity on astrophysical and cosmological scales. Finally, several modified gravity models are introduced, which satisfy the Solar System constrains as well as the constraint on the speed of gravitational waves obtained from almost simultaneous detections of gravitational waves and gamma ray bursts from a neutron star merger (GW170817/GRB 170817A).
\end{abstract}

Keywords: cosmology, general relativity, modified gravity

PACS numbers:95.36.+x

\section{Introduction}

Cosmology has entered the era of precision science. We now have the standard model of cosmology, Lambda Cold Dark $(\Lambda \mathrm{CDM})$ model, which explains various observations from the Cosmic Microwave Background to Large Scale Structure with only 6 cosmological parameters. 1 At the same time, in the $\Lambda \mathrm{CDM}$ model, only $5 \%$ of the energy density of the universe is made of known matter. $25 \%$ of the energy density is made of dark matter while $70 \%$ of the energy density is made of the cosmological constant. The cosmological constant is required to explain the accelerated expansion of the universe but its observed value is many orders of magnitude smaller than what we expect from the standard model of particle physics. This cosmological constant problem motivates us to consider alternatives to the $\Lambda \mathrm{CDM}$ model. In this article, we focus on modifications to general relativity and discuss motivations to consider theories beyond general relativity and explain various constraints that these theories need to satisfy. We pay particular attention to screening mechanisms to evade stringent Solar System constraints while having large modifications on astrophysical and cosmological scales. We then discuss several examples of models that satisfy the Solar System constrains as well as the constraint on the speed of gravitational waves 
obtained by almost simultaneous detections of gravitational waves and gamma ray burst from a neutron star merger (GW170817/GRB 170817A) [2]

Throughout this chapter, we use the metric signature $(-,+,+,+)$.

\section{Lambda Cold Dark matter ( $\Lambda$ CDM) model}

This section introduces the standard $\Lambda$ CDM model, emphasising the basic assumptions of the model, and summarises observational constraints on the model mainly from the Planck 2015 results 1

\section{1. $\Lambda$ CDM model}

The standard model of cosmology is based on three assumptions:

(1) Our Universe is homogeneous and isotropic on average on large scales.

(2) Gravity is described by general relativity on all scales.

(3) The matter content of our universe is given by Cold Dark Matter (CDM), baryons and radiation.

The first assumption implies that the metric describing the background homogeneous and isotropic universe is given by

$$
d s^{2}=-d t^{2}+a(t)^{2}\left[d \chi^{2}+f_{K}(\chi)\left(d \theta+\sin ^{2} \theta d \phi^{2}\right)\right], \quad f_{K}=\frac{1}{\sqrt{-K}} \sinh (\sqrt{-K} \chi),
$$

where $a(t)$ is the scale factor and $K=0,1$ and -1 correspond to flat, closed and open geometry of the three dimensional time constant hypersurface, respectively. The comoving distance is defined as

$$
\chi=-\int_{t_{0}}^{t} \frac{1}{a\left(t^{\prime}\right)} d t^{\prime}=\frac{1}{H_{0}} \int_{0}^{z} \frac{d z^{\prime}}{E\left(z^{\prime}\right)}, \quad E(z)=\frac{H(z)}{H_{0}},
$$

where quantities with the subscript 0 indicates that they are evaluated today at $t=t_{0}, z=1 / a-1$ is the redshift and $H=\dot{a} / a$ is the Hubble function. The luminosity and angular diameter distance are defined as

$$
d_{L}=f_{K}(\chi)(1+z), \quad d_{A}=\frac{d_{L}}{(1+z)^{2}} .
$$

The Hubble parameter today $H_{0}$ plays a key role in dark energy and modified gravity models. In the natural unit, $c=\hbar=k_{B}=1$, it is given as $H_{0}=2.13 \times 10^{-42} h \mathrm{GeV}$ where $h$ is the dimensionless Hubble parameter, which needs to be determined by observations.

The second assumption implies that gravity is described by the Einstein-Hilbert action

$$
S=\frac{1}{16 \pi G} \int d^{4} x \sqrt{-g}(R+2 \Lambda)+\int d^{4} x \mathcal{L}_{m}\left[g_{\mu \nu}\right]
$$


where $G$ is the Newton constant, $R$ is the Ricci curvature scalar and $\Lambda$ is the cosmological constant. The Einstein equations are given by

$$
G_{\mu \nu}+\Lambda g_{\mu \nu}=8 \pi G T_{\mu \nu}
$$

where the energy-momentum tensor is defined as $T^{\mu \nu}=(2 / \sqrt{-g}) \delta S_{m} / \delta g_{\mu \nu}$. The Planck energy is defined as $M_{\mathrm{pl}} \equiv \sqrt{1 / 8 \pi G}=2.43 \times 10^{18} \mathrm{GeV}$. As we will discuss later, we do not expect that general relativity is valid at energy scales higher than $M_{\mathrm{pl}}$ but this is not relevant as long as we consider the late time evolution of the universe. Applying to the metric given by (1), we obtain the Friedmann equations

$$
\begin{aligned}
H^{2} & =\left(\frac{\dot{a}}{a}\right)^{2}=\frac{8 \pi G}{3} \rho-\frac{K}{a^{2}}+\frac{\Lambda}{3}, \\
\frac{\ddot{a}}{a} & =-\frac{4 \pi G}{3}(\rho+3 P)+\frac{\Lambda}{3},
\end{aligned}
$$

where $T_{\nu}^{\mu}=\operatorname{diag}(-\rho, P, P, P), \rho$ is the energy density and $P$ is the pressure of matter. The Ricci curvature scalar is given by $R=6\left(a \ddot{a}+\dot{a}^{2}+K\right) / a^{2}$.

Now we use the final assumption to specify the matter content of the universe. The Friedmann equation gives

$$
E(z)=\Omega_{m}(1+z)^{3}+\Omega_{r}(1+z)^{4}+\Omega_{K}(1+z)^{2}+\Omega_{\Lambda},
$$

where $\Omega_{m}=\Omega_{b}+\Omega_{c}, \Omega_{r}, \Omega_{K}$ and $\Omega_{\Lambda}$ are the contributions from cold dark matter and baryons, radiation, curvature and the cosmological constant, respectively. The contribution from radiation, $\Omega_{r}$, is precisely known from Cosmic Microwave Background $(\mathrm{CMB})$ as $\Omega_{r} h^{2}=2.47 \times 10^{-5}$. The minimum model that fits the CMB data is the flat $\Lambda \mathrm{CDM}$ model, which has 6 parameters, $\Omega_{b} h^{2}, \Omega_{c} h^{2}, \theta_{*}, \tau, A_{s}$ and $n_{s}$, where $\theta_{*}$ is the angular scale of the sound horizon at the last scattering, $\tau$ is the optical depth, $A_{s}$ is the amplitude of the primordial fluctuations and $n_{s}$ is the spectrum index of the power spectrum of the primordial curvature perturbation. The Planck 2015 result ${ }^{11}$ gave the constraint $\Omega_{m}=0.308 \pm 0.012, \Omega_{\Lambda}=0.692 \pm 0.012$ and $H_{0}=67.8 \pm 0.92 \mathrm{~km} / \mathrm{s} / \mathrm{Mpc}$, assuming $\Omega_{K}=0$. Note that the constraint on $H_{0}$ is an indirect one derived from the 6 parameters by marginalising over other parameters. Therefore it highly depends on the assumption of the cosmological model, i.e. the flat $\Lambda \mathrm{CDM}$ model ${ }^{3}$ The curvature is constrained as $\Omega_{K}=-0.005_{-0.017}^{+0.016}$ by the CMB measurement alone although there is a degeneracy between $\Omega_{K}$ and $H_{0}$.

The most notable feature of the $\Lambda \mathrm{CDM}$ model is the large contribution from the cosmological constant. This is required because the expansion of the universe is accelerating rather than decelerating, $\ddot{a}>0$. This was first found by the observations of supernovae $\sqrt[4]{5}$ which measure the luminosity distance $d_{L}$ to supernovae. On the other hand, the acoustic peaks of CMB anisotropies are determined by the sound horizon at radiation drag and the measurements of these acoustic peaks give the information on the angular diameter distance to the last scattering surface. The same Baryon Acoustic Oscillations (BAO) are encoded in the distribution of galaxies and provide the measurement of angular diameter distances at low redshifts from 
galaxy surveys $6 / 7$ These measurements are complimentary in the constraints of $\Omega_{m}$ and $\Omega_{\Lambda}$. The $\Lambda \mathrm{CDM}$ model is currently consistent with all these measurements.

Finally, we mention some tension in the current data with $\Lambda \mathrm{CDM}$. The late time measurements of clustering such as Sunyaev-Zeldovich effects and weak lensing prefer lower amplitudes of the matter fluctuations compared with the Planck measurements of the initial amplitude at last scattering in $\Lambda \mathrm{CDM} 18010$ The most notable tension is the measurements of the Hubble constant $H_{0}$. The local measurements using the distance ladder prefer higher $H_{0}$ than the Planck measurements. For example, the Hubble Space Telescope (HST) measurements give $H_{0}=73.45 \pm 1.66$ $\mathrm{km} / \mathrm{s} / \mathrm{Mpc}, \frac{11}{11}$ which is inconsistent at the 3.5 sigma level with Planck $\Lambda$ CDM. Note that the recent gravitational wave detection with almost simultaneous detections of short gamma ray bursts from a neutron star merger gave an independent measurement of $H_{0}, H_{0}=70_{-8.0}^{+12.0} \mathrm{~km} / \mathrm{s} / \mathrm{Mpc}^{[12}$

It still needs to be seen whether these discrepancies are due to unknown systematics or not. However, if they persist in the future measurements, this may be an indication of new physics beyond $\Lambda$ CDM.

\subsection{Cosmic acceleration}

Although the current observational data is consistently explained by the $\Lambda$ CDM model, it requires the existence of the cosmological constant. The energy scale of the cosmological constant is given by

$$
\rho_{\Lambda} \equiv \frac{\Lambda}{8 \pi G} \sim\left(10^{-3} \mathrm{eV}\right)^{4} .
$$

Quantum field theory predicts that all particles give rise to vaccum energy $T_{\mu \nu}=$ $-V_{\text {vac }} g_{\mu \nu}$, which contributes to the cosmological constant. The Standard Model of particle physics includes particles up to the $\mathrm{TeV}$ scale and we would estimate the vacuum energy to be $V_{\text {vac }}>(\mathrm{TeV})^{4}$. This is 60 orders of magnitude larger than the cosmological constant that we need to explain the current observations. This is known as the (old) cosmological constant problem, i.e. why the vacuum energy does not gravitate as expected according to Einstein's theory of gravity 13 This problem existed even before the discovery of the accelerated expansion of the universe. The discovery of the accelerated expansion has created a new cosmological constant problem, i.e. the coincident problem - why does the expansion of the universe start to accelerate just now? This requires that the cosmological constant is fine-tuned to be given by $(9)$.

There have been many attempts to explain the observed accelerated expansion of the universe. Note that in most of these approaches, the old cosmological constant problem is assumed to be solved by some mechanism. There are three main approaches depending on which assumption of the $\Lambda \mathrm{CDM}$ model to abandon.

The first possibility is to consider an inhomogeneous universe. Indeed, our universe is inhomogeneous, otherwise we do not exist. If the formation of inhomogeneous structures causes a back reaction to the expansion of the universe and it 
causes the expansion of the universe to accelerate, we can solve the coincident problem as the onset of the acceleration coincides with the formation of first non-linear structures. The back reaction is a notoriously difficult problem in general relativity due to the non-linear nature of Einstein's equations. There are still on-going debates on the magnitude of back-reaction ${ }^{14}[15$ but it is a general consensus that it is likely not large enough to explain the current acceleration of the universe. Another related possibility is to abandon the Copernican principle and assume that we are living in a special place in the universe such as a centre of a large void. Although it is relatively easy to explain supernavae observations in this model, it is not easy to reconcile this model with all other observations such as kinetic Sunyaev-Zel'dovich effects ${ }^{[16}$ Note that even if inhomogeneity cannot explain the accelerated expansion, it can still play an important role in precise cosmological measurements.

The second possibility is that general relativity is modified on large scales. See reviews. 17 .19] As we will see below, general relativity has been tested in the Solar System to high accuracy. It successfully predicts the orbital decay of the HulseTaylor binary pulsar. ${ }^{20}$ Recently the prediction of gravitational waves has been confirmed by direct detections of gravitational waves from binary black holes ${ }^{21}$ as well as a binary neutron star. ${ }^{22}$ However, all these constraints on deviations from general relativity apply to scales that are vastly different from scales accessible by cosmological and astrophysical measurements. Thus it is still possible that large distance modifications of gravity can account for the accelerated expansion of the universe.

Finally, the acceleration can be caused by unknown dark energy. The distinction between dark energy and modified gravity is generally ambiguous. Indeed, in the background, any modification of gravity or dark energy can be described as the existence of an effective energy density $\rho_{\text {dark }}$ and pressure $P_{\text {dark }}$. The only parameters required to describe the background expansion are the equation of state $w_{\text {dark }}(z)=P_{\text {dark }} / \rho_{\text {dark }}$ and the density parameter $\Omega_{\text {dark }}$. The background expansion can be parametrised as

$E(z)=\Omega_{m}(1+z)^{3}+\Omega_{r}(1+z)^{4}+\Omega_{K}(1+z)^{2}+\Omega_{\mathrm{dark}} \exp \left(3 \int_{0}^{z} d z^{\prime} \frac{1+w_{\mathrm{dark}}\left(z^{\prime}\right)}{1+z^{\prime}}\right)$.

The Planck 2015 gave a constraint on a constant equation of state as $w_{\text {dark }}=$ $-1.006 \pm 0.045$. The time dependence of $w_{\text {dark }}(z)$ is a clear evidence that the acceleration is not caused by the cosmological constant. A recent attempt to combine various data sets indicate that the evolving $w_{\text {dark }}$ model is preferred at a $3.5 \sigma$ significance level based on the improvement in the fit although the Bayesian evidence for the dynamical $w_{\text {dark }}$ model is insufficient to favour it over $\Lambda \mathrm{CDM} \cdot 23$

If we consider the formation of structure, there is a difference between simple dark energy models and modified gravity models. Here simple dark energy models mean that dark energy does not cluster and it has no effects on structure formation. This is the case in quintessence models where dark energy is described by a 
scalar field with the standard kinetic term $\sqrt[24]{25}$ In these models, the inhomogeneity of the scalar field can be ignored under horizon scales. In the simple dark energy models, the only parameters of the model are the equation of state $w_{\text {dark }}$ and the density parameter $\Omega_{\mathrm{dark}}$. Thus structure formation is completely determined by the background evolution of the universe. While in modified gravity models, this connection is generally lost. Thus the combination of measurements of the background expansion and structure formation offers a way to distinguish between these two scenarios. See Ref ${ }^{[26}$ for discussions on the distinction between dark energy and modifed gravity models.

\section{Modified gravity models}

In this review, we mainly focus on modified gravity models. We will first discuss motivations to consider modifications to general relativity. Then we discuss various observational constraints as well as theoretical constraints that any modified gravity models need to satisfy.

\subsection{Motivations}

The discovery of cosmic acceleration has renewed interests to study theories of modified gravity. There are mainly four motivations to consider modified gravity theories.

(1) Quantum gravity

General relativity is not a renormalisable theory as is clear from the fact that the coupling constant $G$ has a mass dimension of -2 . Thus general relativity is thought as an effective theory that is valid up to the Planck scale $M_{\mathrm{pl}}$ where quantum gravity effects become important. There are many attempts to construct quantum theory of gravity such as string theory but the complete quantum theory of gravity is still out of our reach. An important lesson that we have learnt is that the Planck scale $M_{\mathrm{pl}}$ is not necessary the scale at which general relativity is modified and the quantum gravity scale can be much lower. For example, there has been an attempt to consider a model where the fundamental scale of gravity is $\mathrm{TeV}$ to solve the hierarchy between the electroweak scale and the Planck scale.27

(2) The cosmological constant problem

One of the reasons to modify gravity is to explain why we do not see large vacuum energy expected from the Standard Model of particle physics. One possible solution is that the vacuum energy does not gravitate. This is known as degravitation or self-tunning. There is a no-go theorem by Weinberg that forbids self-tunning solutions under several assumptions ${ }^{13}$ and any attempts need to evade this no-go theorem. A simple example is a braneworld model in six dimensions ( $\mathrm{se}^{28}$ for a review). In this model, the standard model particles are confined to a four dimensional brane in six dimensions with two extra spatial 
dimensions. The cosmological constant in the four dimensional brane does not change the geometry of the four dimensional spacetime and it only curves the extra two dimensional space. The challenge is that it is difficult to modify gravity only for the cosmological constant while reproducing general relativity for normal matter. See the reference ${ }^{29}$ for a review and references therein.

(3) Cosmic acceleration

Modified gravity models provide a possibility to realise the accelerated expansion of the universe without the cosmological constant. A simply example is provided by a five dimensional braneworld model proposed by Dvali, Gabadadze and Porrati (DGP) ${ }^{301}$ In this model the Friedman equation on a four-dimensional brane is given by $H^{2}=H / r_{c}+8 \pi G \rho / 3$ where $r_{c}$ is defined as a ratio between four-dimensional and five-dimensional Newton constant. Even if the energy density $\rho$ does not contain the cosmological constant, the expansion of the universe accelerates as the Hubble parameter approaches constant, $H \rightarrow 1 / r_{c}$, at late times. This is called self-acceleration. Unfortunately, this particular solution suffers from instabilities $\$ 31]$ but the idea of self-acceleration has been studied intensively in many other modified gravity models.

(4) Tests of general relativity

General relativity has been tested exquisitely in the Solar System and by binary pulsars ${ }^{33}$ However, this does not imply that general relativity is valid on all scales in any environments. It is worth pursuing the tests of general relativity on different scales and in different environments to understand the validity regime of general relativity. For example, the recent detections of gravitational waves have opened a new window to test gravity in strong gravity regime as well as to test a propagation of gravitational waves. In the next decade, a number of cosmological surveys aiming to reveal the nature of cosmic acceleration will produce the data. These measurements can be used to test general relativity on cosmological scales. To satisfy stringent Solar System constraints, modified gravity theories often incorporate screening mechanisms to hide modifications of gravity in dense environments. These screening mechanisms provide novel ways to test deviations from general relativity. For example, screening mechanisms can introduce environmental dependent modifications of gravity, which can be tested using astrophysical measurements. Individual atoms inside a large enough high-vacuum chamber can be unscreened, giving a possibility to detect modifications of gravity in laboratory tests. These novel tests of gravity are one of the main focuses of this special issue.

\subsection{Observational constraints}

Modified gravity models need to satisfy various observational constraints. Here we list several important constraints that will be used later. See the reference ${ }^{33}$ for a comprehensive review.

(1) Solar system tests 
First conditions come from the Solar System tests. The Parametrised Post Newtonian $(\mathrm{PPN})$ metric is given by

$$
d s^{2}=-\left(1-2 U+2 \beta U^{2}\right) d t^{2}+(1-2 \gamma U) \delta_{i j} d x^{i} d x^{j},
$$

where $U=\int d^{3} x^{\prime} \rho\left(x^{\prime}\right) /\left|\mathbf{x}-\mathbf{x}^{\prime}\right|$. The time dilation due to the effect of the Sun's gravitational field was measured very accurately using the signal from Cassini satellite. This gives the constraint on $\gamma$ as

$$
\gamma-1<(2.1 \pm 2.3) \times 10^{-5} .
$$

The constraint on $\beta$ comes from the perihelion shift of Mercury. Assuming the Cassini bound, the constraint on $\beta$ is given by $\beta-1=(-4.1 \pm 7.8) \times 10^{-5}$.

(2) Lunar Laser Ranging

The Lunar Laser Ranging (LLR) experiment gives various constraints ${ }^{34}$ It gives tight constraints on the deviations of gravitational potential from the general relativity prediction. The anomalous perihelion angular advance of the Moon is constrained as

$$
|\delta \theta|=\left|\pi r \frac{d}{d r}\left[r^{2} \frac{d}{d r}\left(\frac{\varepsilon}{r}\right)\right]\right|<2.4 \times 10^{-11}, \quad \varepsilon=\frac{\delta \Psi}{\Psi},
$$

where $\varepsilon$ is the radial dependent deviation of the gravitation potential $\Psi=$ $-G M / r$. The LLR experiments also constrain the time variation of the Newton constant

$$
\frac{\dot{G}}{G}=(2 \pm 7) \times 10^{-13} .
$$

Finally, the LLR provides precision tests of the weak equivalence principle. The difference between the acceleration of the Earth and the Moon is constrained as

$$
\eta \equiv \frac{2\left|a_{\text {earth }}-a_{\text {moon }}\right|}{a_{\text {earth }}+a_{\text {moon }}}<10^{-13} .
$$

(3) Hulse-Taylor binary pulsar

The orbital decay of the Hulse-Taylor binary pulsar due to gravitational wave emissions is consistent with the general relativity prediction. ${ }^{35}$ After correcting the effect of a relative acceleration between the binary pulsar system and the Solar System caused by the differential rotation of the galaxy, the observed rate of change of orbital period compared with the general relativity prediction is given by

$$
\frac{\dot{P}}{\dot{P}_{\mathrm{GR}}}=0.997 \pm 0.002 .
$$

(4) Gravitational wave propagation

On 17 August 2017, gravitational waves from a neutron star merger were detected by LIGO ${ }^{22}$ Almost simultaneously, short gamma ray bursts were detected. The observed time delay was $(+1.74 \pm 0.05)$ s. This put stringent constraints on the difference between the speed of gravitational waves $c_{\mathrm{GW}}$ and the 
speed of light $c$ as ${ }^{2}$

$$
-3 \times 10^{-15}<\frac{c_{\mathrm{GW}}}{c}-1<7 \times 10^{-16} .
$$

The lower bound was obtained assuming that the short gamma ray bursts signal was emitted $10 \mathrm{~s}$ after the GW signal. This constraint applies to a local universe. The distance to the neutron star merger is estimated as $43.8_{-6.9}^{+2.9} \mathrm{Mpc}$. In terms of redshift, this corresponds to $z \sim 0.01$. Note that there is also a lower bound $-2 \times 10^{-15}<c_{\mathrm{GW}} / c-1$ from the gravitational Cherenkov radiation emitted by high energy cosmic rays if the speed of gravitational waves is smaller than the speed of light $\frac{36}{36}$ This constraint has a significant implication to many modified gravity model $\sqrt{37}[43$ as we will see later.

\subsection{Theoretical conditions}

Another condition that modified gravity models need to satisfy is the theoretical consistency of the model. Modified gravity models often introduce additional degrees of freedom as we discuss below and these additional modes can lead to instabilities. To illustrate several types of instabilities, let us consider a simple scalar field described by the action

$$
S=\int d t d^{3} x\left(K_{t} \dot{\phi}^{2}-K_{x}\left(\partial_{i} \phi\right)\left(\partial^{i} \phi\right)-m^{2} \phi^{2}\right) .
$$

The tachyonic instability arises when the scalar field has a negative mass squared $m^{2}<0$. Whether this instability is catastrophic or not depends on the instability time scale determined by the mass as the time scale of the instability is given by $\left|m^{2}\right|^{-1 / 2}$. Another instability arises when the gradient term has a negative coefficient $K_{x}<0$. In this case, the instability time scale is determined by the wave number of the mode, so the time scale becomes shorter on smaller scales. Finally, the ghost instability arises when the time kinetic term of the scalar field has a wrong sign $K_{t}<0$. At the quantum level, the vacuum is unstable as negative energy particles can be created from vacuum and it decays instantaneously. To avoid the instability, it is required to introduce a non-Lorentz-invariant cut-off in the theory (sec ${ }^{44}$ for a review).

Another problem is known as the strong coupling problem, which arises from non-linear interaction terms. An example of the non-linear term that appears later is 31

$$
S_{\text {non-linear }}=-\int d^{4} x \frac{1}{\Lambda_{3}^{3}} \square \phi(\partial \phi)^{2} .
$$

This non-linear interaction is suppressed by $\Lambda_{3}$. Quantum corrections generate other terms suppressed by $\Lambda_{3}$ such as $S \propto-\left(1 / \Lambda_{3}^{6}\right) \int d^{4} x \phi \square^{4} \phi$, and we lose control of the theory beyond $\Lambda_{3}$. This strong coupling scale is often associated with the energy scale related to the accelerated expansion of the Universe, $H_{0}$, which is extremely small compared with the scale of gravity $M_{\mathrm{Pl}}$. Thus we will often find that the 
strong coupling scale is rather low in modified gravity models. This means that we need to treat these theories as an effective theory, which is valid only at energy scales lower than $\Lambda_{3}$. It is an interesting question whether there exists a standard (i.e. local, unitary, analytic and Lorentz-invariant) Ultra-Violet (UV) completion of these theories $\frac{45}{4}$ The condition to have a standard UV completion restricts the parameter space of effective theory (see for example ${ }^{\sqrt{46}}$ ).

\subsection{Classifications}

There are numerous modified gravity models proposed in the literature. To classify these models in a general framework, Lovelock's theorem plays an important role. Lovelock's theorem proves that Einstein's equations are the only second-order local equations of motion for a single metric derivable from the covariant action in fourdimensional spacetime. This indicates that if we modify general relativity we need to violate one or more of the assumptions in Lovelock theorem.

(1) Higher derivatives

We normally require that the equations of motion contain up to second time derivatives. The Ostrogradsky theorem states that higher order time derivatives introduce additional degrees of freedom, which makes the Hamiltonian unbounded from below. This can be shown by introducing a new variable for a higher derivative term. This theorem applies if all the conjugate momenta including those associated with higher derivatives can be expressed in terms of velocities and variables. This is called the non-degeneracy condition. If this non-degeneracy condition is not satisfied, it is possible to have higher derivative theories without introducing instabilities ${ }^{47}$ A typical example is $f(R)$ gravity models, which contain fourth order time derivatives of metric in the equations of motion but do not propagate the Ostrogradsky ghost. Another example is degenerate higher order scalar tensor theories $\frac{48}{6}$ We will review these theories below.

(2) Non-locality

An example of non-local gravity is to modify Einstein-Hilbert action to $R f\left(\square^{-1} R\right)$ where $\square^{-1}$ is the inverse Laplacian operator $49 \sqrt[40]{50}$ The main advantage of this theory is that $\square^{-1} R$ has no dimension so in principle it is possible to avoid the introduction of the fine-tuned mass dimension. Another example is $m^{2} R \square^{-2} R{ }^{51}$ The meaning of the non-local operator $\square^{-1}$ needs to be defined carefully. In the equations of motion, these operators are understood as the retarded Green function in order to preserve causality. This cannot be obtained from a classical action thus these actions should be considered as a quantum effective action. These theories can be localised by introducing scalar fields. In fact, the $m^{2} R \square^{-2} R$ theory can be written as a bi-scalar tensor theory. One of the scalar field has a ghost-like kinetic term but it is argued that there is no quanta associated with this ghost field. See the reference ${ }^{52}$ for details. 
(3) Higher dimensional spacetime

Another radical idea is to consider higher dimensional spacetime. There are stringent constraints on the existence of extra-dimensions for standard model particle interactions. These constraints are normally avoided by compactifying the extra-dimensions. In this case, modifications of gravity only appears on length scales shorter than the size of extra-dimensions. Another possibility is to consider a brane on which standard model particles are confined. We will review a braneworld model that admits long distance modifications of gravity.

(4) Lorentz violation

A typical example of Lorentz non-invariant theory is Einstein-Aether theory. In this theory, a vaccum expectation value of a vector field breaks Lorentz invariance. Lorentz non-invariant theory plays a role in the construction of renormalisable theory of gravity. By admitting higher spatial derivatives, it is possible to improve the UV behaviour of gravity as in Horava gravity. These theories are tightly constrained by various tests of gravity, most notably the constraints on the deviation of the gravitational wave speed from the speed of light. See the references $53 \sqrt[54]{5}$ for details.

(5) Extra degrees of freedom

In additional to metric, additional degrees of freedom such as scalar fields, vector fields or tensor fields can be introduced. Scalar tensor theories are one of the most well studied modified gravity models where a scalar field is non-minimally coupled to gravity. Other well studied theories are massive gravity and bi-gravity theories. In massigve gravity, a fiducial metric is introduced while in bi-gravity models, an additional dynamical metric is introduced. See the referenc ${ }^{55}$ for a review.

\section{Screening mechanisms}

In the previous section, we classify various modified gravity models. Many of these models introduce an additional scalar degree of freedom. For example, five dimensional gravitons and massive gravitons in four-dimensions both have five degrees of freedom. One of them is a helicity- 0 mode, which behaves as a scalar field on small scales.

If this scalar degree of freedom couples to matter, it is subject to stringent constraints from the Solar System tests. Screening mechanisms provide a way to evade this condition and they are included in many well-studied modified gravity models. See the references $\sqrt{18] 19}$ for reviews and references therein.

\subsection{Motivations}

In order to understand the need for screening mechanisms, let us consider BransDicke gravity described by the action

$$
S=\frac{1}{16 \pi G} \int d^{4} x \sqrt{-g}\left(\psi R-\frac{\omega_{\mathrm{BD}}}{\psi}(\partial \psi)^{2}\right)+S_{m}\left[g_{\mu \nu}\right] .
$$


We are interested in forces generated by a non-relativistic source $T_{0}^{0}=-\rho$. Using the quasi-static approximations to neglect time derivatives, the perturbations of the metric

$$
d s^{2}=-(1+2 \Psi) d t^{2}+(1-2 \Phi) \delta_{i j} d x^{i} d x^{j},
$$

and the scalar field perturbation $\psi=1+\varphi$ obey the following equations

$$
\begin{array}{r}
\nabla^{2} \Psi=4 \pi G \rho-\frac{1}{2} \nabla^{2} \varphi, \\
\left(3+2 \omega_{\mathrm{BD}}\right) \nabla^{2} \varphi=-8 \pi G \rho, \\
\Phi-\Psi=\varphi .
\end{array}
$$

From these equations, we observe that the scalar field perturbation $\varphi$ gives an additional contribution to the Poisson equation. This introduces an additional force, often called the fifth force. The scalar field perturbation also changes the relation between the two metric perturbations $\Phi$ and $\Psi$. The solutions for the metric perturbations are given by

$$
\nabla^{2} \Psi=4 \pi G \mu \rho, \quad \Psi=\eta^{-1} \Phi
$$

where

$$
\mu=\frac{4+2 \omega_{\mathrm{BD}}}{3+2 \omega_{\mathrm{BD}}}, \quad \eta=\frac{1+\omega_{\mathrm{BD}}}{2+\omega_{\mathrm{BD}}} .
$$

We recover general relativity in the large $\omega_{\mathrm{BD}}$ limit. Indeed, imposing the Solar System constraints 12, , we obtain $|\eta-1|=(2.1 \pm 2.3) \times 10^{-5}$. Then the constraint on $\omega_{\mathrm{BD}}$ is given by $\omega_{\mathrm{BD}}>40,0000^{33}$ Once we impose this constraint on the parameter of model $\omega_{\mathrm{BD}}$, the deviations from general relativity is suppressed, i.e. $|\mu-1|<10^{-5}$ and $|\eta-1|<10^{-5}$, on all scales.

\subsection{Classifications}

In order to avoid this blanket suppression of deviations from general relativity on all scales, the Brans-Dicke theory needs to be extended to include non-linear functions of the scale field. Schematically, the generalised action is given by

$S=\frac{1}{16 \pi G} \int d^{4} x \sqrt{-g}\left(\psi R-\frac{\omega(\psi)}{\psi}(\partial \phi)^{2}+\mathcal{K}\left[(\partial \psi)^{2},\left(\partial^{2} \psi\right)\right]-2 U(\psi)\right)+S_{m}\left[g_{\mu \nu}\right]$,

where $\mathcal{K}$ is a function of first and second derivative of the scalar field. This function needs to be chosen carefully not to introduce the Ostrogradsky ghost. We will give examples of these functions later.

The screening mechanisms can be classified formally depending on which nonlinear function in (27) is used to suppress the fifth force.

(1) Chameleon mechanism $56 / 57$

In this mechanism, the potential $U(\psi)$ is introduced. The scalar field takes 
a background value $\psi_{\mathrm{BG}}$ determined by the background density. The potential introduces a mass term for the scalar field perturbation around this background

$$
\left(3+2 \omega_{B D}\right) \nabla^{2} \varphi+m\left(\psi_{\mathrm{BG}}\right)^{2} \varphi=-8 \pi G \rho,
$$

where the mass depends on the background field. The scalar field does not propagate beyond the Compton wavelength $m^{-1}$ as the solution decays as $\exp (-m r)$ where $r$ is the distance from the source. Since the mass depends on the background density, it is possible to realise a situation where the mass is large in dense environments such as the Solar System while it is small on a cosmological background, modifying gravity non-trivially.

(2) Dilaton ${ }^{58}$ and Symmetron mechanism 59

The coupling of the scalar field to matter is determined by the function $\omega(\psi)$, which is determined by the background scalar field $\psi_{\mathrm{BG}}$ thus depends on a background density. It is possible to consider a model where $\omega\left(\psi_{\mathrm{BG}}\right)$ is large in dense environments so that the scalar field decouples from matter while $\omega$ is $\mathcal{O}(1)$ on cosmological background.

(3) K-mouflage ${ }^{60}$ and Vainshtein mechanism ${ }^{61}$

In this case, the non-linear kinetic term $\mathcal{K}\left[(\partial \psi)^{2},\left(\partial^{2} \psi\right)\right]$ plays a role to effectively suppress the coupling between the scalar field and matter. K-mouflage mechanism uses the non-linearity of the first derivative of the scalar field while the Vainshtein mechanism relies on the non-linearity of the second derivative of the scalar field in the action.

These screening mechanisms can be classified in another way depending on how it operates to suppress the fifth force for a spherically symmetric object with a mass $M$ and size $R !^{18}$

(1) Thin-shell screening

In this category of models, screening is determined by the gravitational potential of the object $|\Psi|=G M / R$. The condition for screening is given by the thinshell condition $\sqrt[56]{57}$ which requires that the gravitational potential is larger than a critical value set by the background scalar field $\psi_{\mathrm{BG}},|\Psi|>\chi_{\mathrm{BG}}\left(\psi_{\mathrm{BG}}\right)$. Chameleon, Dilaton and Symmetron screening belong to this type of screening mechanism.62

(2) K-mouflage mechanism

In this case, screening is determined by the first derivative of the gravitational potential $|\partial \Psi| \sim G M / R^{2}$. The screening happens if this exceeds a critical mass scale set by a model parameter, $|\partial \Psi|>\Lambda_{c}$.

(3) Vainshtein mechanism

Screening is determined by the second derivative of the gravitational potential $\left|\partial^{2} \Psi\right| \sim G M / R^{3}$. This is the three-dimensional curvature generated by the object. Screening operates if the curvature of the object exceeds a critical mass scale squared set by a model parameter, $\left|\partial^{2} \Psi\right|>\Lambda_{c}^{2}$. 


\subsection{Frame transformation}

The screening mechanisms that utilise non-linear functions of the scalar field, $\omega(\psi)$ and $U(\psi)$, are described by generalised Brans-Dicke theory described by the action

$$
S=\frac{1}{16 \pi G} \int d^{4} x \sqrt{-g}\left(\psi R-\frac{\omega(\psi)}{\psi}(\partial \psi)^{2}-2 U(\psi)\right)+S_{m}\left[g_{\mu \nu}\right]
$$

Chameleon, dilaton and symmetron mechanisms can be implemented in this theory. This action 29) is formulated in the Jordan frame where matter is coupled to metric $g_{\mu \nu}$ minimally. In order to understand the dynamics of the scalar field, it is useful to define the Einstein frame in which the scalar field is minimally coupled to gravity instead. This can be achieved by a conformal transformation

$$
g_{\mu \nu}^{E}=\psi g_{\mu \nu}
$$

By this transformation, the action becomes

$$
S^{E}=\int d^{4} x \sqrt{-g^{E}}\left[\frac{1}{16 \pi G} R^{E}-\frac{1}{2}(\nabla \phi)^{2}-V(\phi)\right]+S_{m}\left[A^{2}(\phi) g_{\mu \nu}^{E}\right],
$$

where

$$
\left(\frac{d \phi}{d \psi}\right)^{2}=\frac{1}{16 \pi G} \frac{3+2 \omega}{\psi^{2}}, \quad A(\phi)=\psi^{-1 / 2}, \quad V(\phi)=\frac{U(\psi)}{8 \pi G \psi^{2}} .
$$

In this frame, matter is coupled to the scalar field through the effective metric $A^{2}(\phi) g_{\mu \nu}^{E}$. This changes the geodesic equation.

Let us consider a motion of non-relativistic particles. These particles follow the geodesic of the effective metric $g_{\mu \nu}=A^{2}(\phi) g_{\mu \nu}^{E}$. We consider the weak field limit and expand the scalar filed $\phi=\bar{\phi}+\delta \phi$ where $A(\bar{\phi})=1$. By defining the metric perturbations in the Einstein frame

$$
d s_{E}^{2}=-\left(1+2 \Psi^{E}\right) d t^{2}+\left(1-2 \Phi^{E}\right) \delta_{i j} d x^{i} d x^{j}
$$

the geodesic equation is given by

$$
\ddot{x}=-\nabla \Psi^{E}-\frac{\beta}{M_{\mathrm{pl}}} \nabla \delta \phi, \quad \beta \equiv M_{\mathrm{pl}} \frac{d \ln A}{d \phi}=\sqrt{\frac{1}{2(3+2 \omega)}},
$$

where $\Psi^{E}$ satisfies the standard Poisson equation

$$
\nabla^{2} \Psi^{E}=4 \pi G \rho^{E},
$$

where $\rho^{E}$ is the energy density in the Einstein frame. In the weak field limit, this coincides with the energy density in the Jordan frame since $A(\bar{\phi})=1$. See Ref ${ }^{63}$ for discussions on various definitions of the density. In the following we replace $\rho^{E}$ by $\rho$. On the other hand, in the Jorndan frame, the geodesic equation is unmodified but the Poisson equation is modified (see Eq. (22)

$$
\ddot{x}=-\nabla \Psi, \quad \nabla^{2} \Psi=4 \pi G \rho-\frac{1}{2} \nabla^{2} \varphi .
$$


By using the relation between the gravitational potential in the Jordan frame $\Psi$ and that in the Einstein frame $\Psi^{E}, \nabla \Psi=\nabla \Psi^{E}+\left(\beta / M_{\mathrm{pl}}\right) \nabla \phi$, and the relation between $\varphi$ and $\phi, \nabla \phi=-\left(M_{\mathrm{pl}} / 2 \beta\right) \nabla \varphi$, we can easily check that the trajectories of non-relativistic particles in the weak field limit are the same in the Jordan and Einstein frame. In general, it is possible to show that observational predictions are exactly the same in both frames (see Ref ${ }^{[64}$ and references therein).

The dynamics of the scalar field is easier to analyse in the Einstein frame. In the following, we will omit the subscript $\mathrm{E}$ unless there is an ambiguity. The equation of motion for the scalar field is given by

$$
\square \phi=\frac{d V}{d \phi}+\frac{\beta(\phi) \rho}{M_{\mathrm{pl}}} \equiv \frac{d V_{\mathrm{eff}}}{d \phi},
$$

where we consider non-relativistic matter and the effective potential is defined as

$$
V_{\text {eff }}(\phi)=V(\phi)+\rho \ln A(\phi) .
$$

We see that the function $\beta$ determines the coupling between the scalar field and matter.

So far we assumed the weak equivalence principle, i.e. the scalar field is coupled universally to all matter species. It is possible to break this principle and couple the scalar field differently to different species:

$$
S_{m}=S_{\mathrm{cdm}}\left[A_{\mathrm{cdm}}^{2} g_{\mu \nu}^{E}\right]+S_{\text {baryon }}\left[A_{\text {baryon }}^{2} g_{\mu \nu}^{E}\right]+\ldots
$$

The stringent constraints coming from the Solar System tests can be avoided by assuming $\beta_{\text {baryon }}=0$. These models are often called interacting dark energy models ${ }^{65}$ if we identify the scalar field with dark energy. In this case, dark energy is interacting with dark matter but not baryons. In these models, there is no need to introduce screening mechanisms.

\subsection{Thin shell screening}

In this section, we review screening mechanisms in which screening is determined by the gravitational potential $|\Psi|$. These include chameleon, symmetron and dilaton mechanisms. Chameleons screen the fifth force by having a large mass in dense environments while symmetrons and dilatons screen the fifth force by having a small coupling to matter in dense environments. Despite this difference, the condition under which screening operates is given by the same thin shell condition as we will see below. A comprehensive review on chameleon mechanisms can be found in the reference ${ }^{63}$

\subsubsection{Chameleon mechanism}

A typical choice of $V(\phi)$ and $A(\phi)$ to realise the chameleon mechanism is

$$
V(\phi)=V_{0}+\frac{\Lambda_{\mathrm{ch}}^{\ell+4}}{\phi^{\ell}}, \quad A(\phi)=\exp \left(\frac{\beta \phi}{M_{\mathrm{pl}}}\right),
$$


where $V_{0}$ is a bare cosmological constant, $\Lambda_{\mathrm{ch}}$ is a parameter of the potenital with mass dimension one and $\ell$ is a dimensionless parameter. The minimum of the effective potential depends on the density as

$$
\phi_{\mathrm{BG}}=\left(\frac{\ell M_{\mathrm{pl}} \Lambda_{\mathrm{ch}}^{\ell+4}}{\beta \rho}\right)^{\frac{1}{\ell+1}} .
$$

Around this minimum, the scalar field acquires a mass

$$
m\left(\phi_{\mathrm{BG}}\right)=V_{\mathrm{eff}}^{\prime \prime}\left(\phi_{\mathrm{BG}}\right)=\ell(\ell+1) \Lambda_{\mathrm{ch}}^{\ell+4}\left(\frac{\beta \rho}{\ell M_{\mathrm{pl}} \Lambda_{\mathrm{ch}}^{\ell+4}}\right)^{\frac{\ell+2}{\ell+1}} .
$$

If $\ell>-1$, in high density backgrounds, $\phi_{\mathrm{BG}}$ becomes small and $m\left(\phi_{\mathrm{BG}}\right)$ becomes large, satisfying the condition to realise the chameleon mechanism. Fig. 1 1 shows the behaviour of the effective potential.
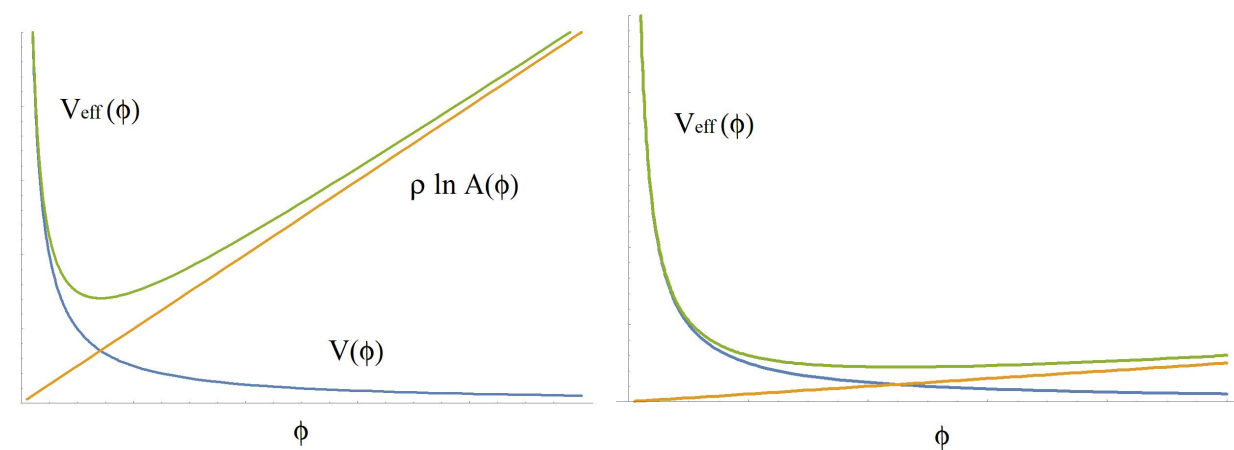

Fig. 1: An example of the potential $V(\phi)$, the contribution from matter $\rho \ln A(\phi)$ and the effective potential $V_{\text {eff }}(\phi)$ in chameleon models. The left panel shows the situation with high density while the right panel shows the low density case.

In order to understand how the chameleon mechanism operates, we consider a spherical object with density $\rho_{s}$ of the size $R$ embedded in a constant density $\rho_{\mathrm{BG}} \ll \rho_{s}$. At infinity, we set the value of the scalar field at the minimum of the potential, $\phi=\phi_{\mathrm{BG}}$ set by $\rho_{\mathrm{BG}}$. Away from the source, perturbations around the background $\phi=\phi_{\mathrm{BG}}+\varphi$ satisfies

$$
\frac{1}{r^{2}} \frac{d}{d r}\left(r^{2} \frac{d \varphi}{d r}\right)=m\left(\phi_{\mathrm{BG}}\right)^{2} \varphi .
$$

The solution for this equations is given by $\varphi(r)=-(C / r) \exp \left[-m\left(\phi_{\mathrm{BG}}\right) r\right]$ where $C$ is an integration constant. On the other hand, inside the source, the scalar field is trapped at the minimum of effective potential $\phi=\phi_{s}$ determined by $\rho_{s}$ between 
$r=0$ and $r=r_{\mathrm{scr}}$. Between $r_{\mathrm{scr}}$ and $R$, the scalar field rolls from $\phi_{s}$ to $\phi_{\mathrm{BG}}$. By matching these solutions, we can determine the integration constants $C$ and $r_{\text {scr }}$. The solution outside the source $r>R$ is given by

$$
\phi(r)=-\left(\frac{3 \Delta R}{R}\right) \frac{2 G M \beta}{r} e^{-m\left(\phi_{\mathrm{BG}}\right) r}+\phi_{\mathrm{BG}},
$$

where

$$
\frac{3 \Delta R}{R}=\frac{3\left(R-r_{\mathrm{scr}}\right)}{R}=\frac{\phi_{\mathrm{BG}}-\phi_{s}}{2 \beta M_{\mathrm{pl}}|\Psi|},
$$

where $|\Psi|=G M / R$. This solution exists only when the thin shell condition $3 \Delta R / R \ll 1$ is satisfied. If this condition is not satisfied, the scalar field does not reach $\phi_{s}$ inside the source and the solution is given by (44) with $3 \Delta R / R=1$.

This result can be understood as follows. Due to the large mass of the scalar field, the scalar field is trapped at the minimum of the potential between $r=0$ and $r=r_{\text {scr }}$ and the mass inside this region does not generate a scalar force if the thin shell condition is satisfied. Only the mass in a shell between $r=r_{\text {scr }}$ and $r=R$

$$
\frac{M-M\left(r_{\mathrm{scr}}\right)}{M} \sim 3 \frac{\Delta R}{R}
$$

generate the scalar force where $M\left(r_{\text {scr }}\right)$ is an enclosed mass in the region $0<r<r_{\text {scr }}$ (see Fig. 2). This is the reason why $r_{\text {scr }}$ is called the screening radius.

The thin shell condition can be expressed in terms of the screening parameter

$$
\chi_{\mathrm{BG}} \equiv \frac{\phi_{\mathrm{BG}}}{2 \beta M_{\mathrm{pl}}}
$$

as $\chi_{\mathrm{BG}}<|\Psi|$ given that $\phi_{s} \ll \phi_{\mathrm{BG}}$. Once this condition is satisfied, the scalar field generated by an object with mass $M$ is given by

$$
\frac{\phi(r)}{M_{\mathrm{pl}}}=-\left(\frac{\chi_{\mathrm{BG}}}{|\Psi|}\right) \frac{2 G M \beta}{r} e^{-m\left(\phi_{\mathrm{BG}}\right) r}+\phi_{\mathrm{BG}} .
$$

Recalling that the geodesic equation for a non-relativistic test particle is given by

$$
\ddot{x}=-\nabla \Psi^{E}-\frac{\beta}{M_{\mathrm{pl}}} \nabla \phi,
$$

we define the normal Newtonian force and the fifth force as

$$
F_{N}=-\nabla \Psi^{E}, \quad F_{\phi}=-\frac{\beta}{M_{\mathrm{pl}}} \nabla \phi .
$$

Using the solution for the scalar field, the ratio of the scalar to Newtonian force outside a source (but within the Compton wavelength) is given by

$$
\frac{F_{\phi}}{F_{N}}=2 \beta^{2} \min \left\{\left(\frac{\chi_{\mathrm{BG}}}{|\Psi|_{i}}\right), 1\right\} \text {. }
$$




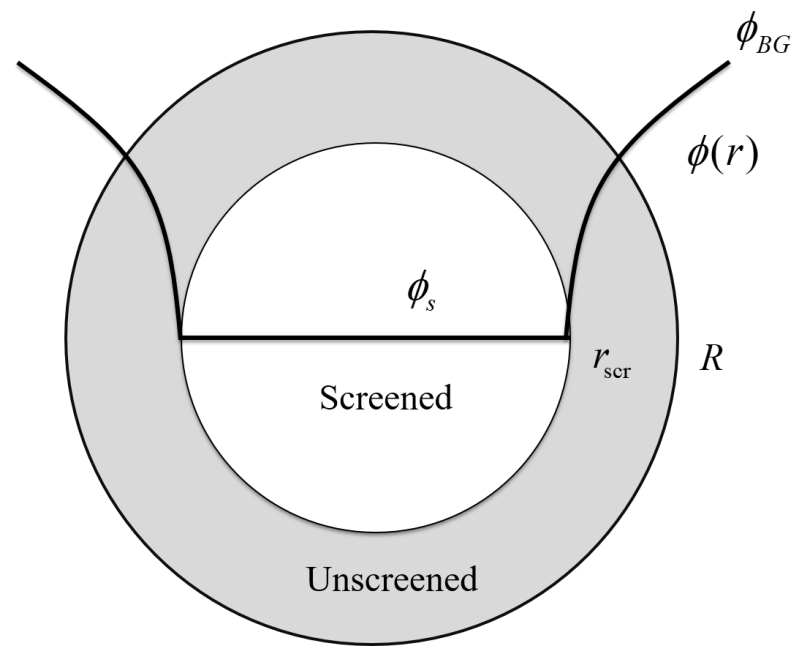

Fig. 2: This figure shows how the thin shell screening works for an object with the size $R$. The scalar field is trapped at the minimum of the effective potential determined by the density of the object, $\phi_{s}$, which is much smaller than the background scalar filed, $\phi_{\mathrm{BG}}$. The mass inside $r=r_{\mathrm{scr}}$ does not produce the scalar force and only the shell between $r=r_{\text {scr }}$ and $r=R$ contribute to the fifth force, suppressing the ratio of the scalar to Newtonian force.

\subsubsection{Symmetron/dilaton mechanism}

A typical choice of $A(\phi)$ and $V(\phi)$ for the symmetron mechanism is given by

$$
V(\phi)=V_{0}-\frac{1}{2} \mu^{2} \phi^{2}+\frac{\lambda}{4} \phi^{4}, \quad A(\phi)=1+\frac{\phi^{2}}{2 M_{s}^{2}},
$$

so that the effective potential becomes

$$
V_{\text {eff }}=-\frac{1}{2} \mu^{2}\left(1-\frac{\rho}{\mu^{2} M_{s}^{2}}\right) \phi^{2}+\frac{\lambda}{4} \phi^{4}
$$

It is easy to see that there is a critical density $\rho_{*}=\mu^{2} M_{s}^{2}$. For high densities, $\rho>\rho_{*}$, the symmetry is restored and the scalar field stays at the minimum $\phi_{\min }=0$ (see Fig. 3). At this minimum, $\beta\left(\phi_{\min }=0\right)=0$ and the scalar field does not couple to matter. On the other hand, for low densities, $\rho<\rho_{*}$, the scalar field evolves to the minimum

$$
\phi_{\min } \sim \pm \frac{\mu}{\sqrt{\lambda}}
$$

Around this minimum, the coupling to matter is given by

$$
\beta\left(\phi_{\min }\right)=\left|\frac{M_{\mathrm{pl}} \phi_{\min }}{M_{s}^{2}}\right| \sim \frac{\mu M_{\mathrm{pl}}}{\sqrt{\lambda} M_{s}^{2}} .
$$




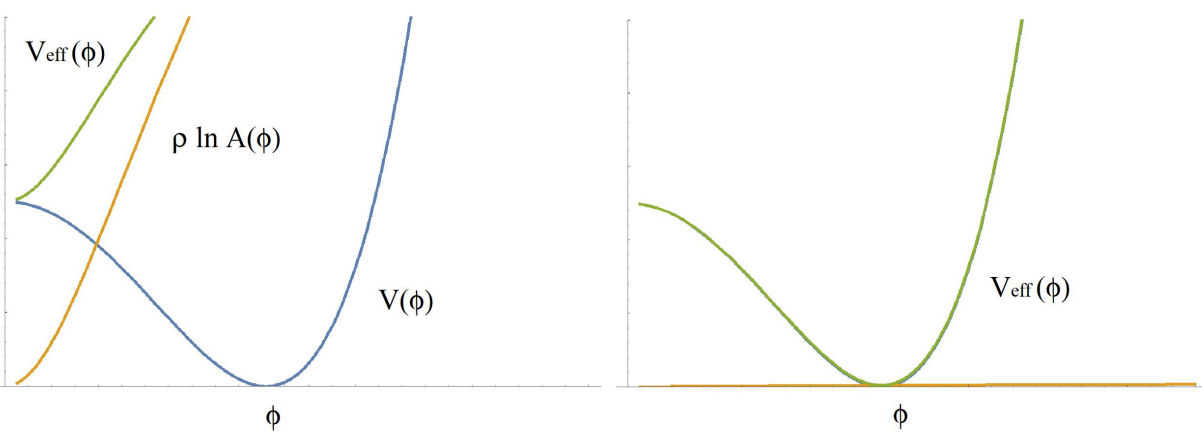

Fig. 3: An example of the potential $V(\phi)$, the contribution from matter $\rho \ln A(\phi)$ and the effective potential $V_{\text {eff }}(\phi)$ in symmetron models. The left panel shows the case with high density, in which the symmetry is restored by the matter contribution and the minimum is given by $\phi_{\min }=0$. The right panel shows the case with low density

Another related mechanism is dilaton. A typical choice of $V(\phi)$ and $A(\phi)$ is

$$
V(\phi)=V_{0} \exp \left(-\frac{\lambda \phi}{M_{\mathrm{pl}}}\right), \quad A(\phi)=1+\frac{A_{2}}{2 M_{\mathrm{pl}}^{2}} \phi^{2} .
$$

The minimum of the effective potential is given by

$$
\phi_{\min }=\frac{\lambda V_{0} M_{\mathrm{pl}}}{A_{2} \rho},
$$

where we assumed $\lambda \phi / M_{\mathrm{pl}} \ll 1$. At the minimum, the coupling constant is given by $\beta=A_{2} \phi / M_{\mathrm{pl}}=\lambda\left(V_{0} / \rho\right)$. As in the symmetron case, for high densities, the minimum of the potential is driven to $\phi_{\min }=0$ thus the coupling of the scalar to matter vanishes, $\beta=0$, while the coupling can be $O(1)$ when the density becomes low compared to $\lambda V_{0}$.

Although the mechanism to suppress the fifth force is different from chameleons, it was shown that the screening condition in these mechanisms is similar ${ }^{[62}$ If we consider a spherically symmetric object, the ratio of the scalar to Newtonian force is given in terms of the screening parameter $\chi_{\mathrm{BG}}$ (47) as in chameleons where $\beta$ is given by $\beta_{\mathrm{BG}} \equiv \beta\left(\phi_{\mathrm{BG}}\right)$.

In the case of symmetrons, domain walls can be formed after the phase transition. This requires the study of full time dependent dynamics of the scalar field. This was performed with N-body simulations and it was shown that domain walls do form but their effects on the distribution of matter are small.66 


\subsubsection{Thin shell screening}

In all above screening mechanisms, the thin shell condition can be expressed in terms of the self-screening parameter

$$
\chi_{\mathrm{BG}} \equiv \frac{\phi_{\mathrm{BG}}}{2 \beta_{\mathrm{BG}} M_{\mathrm{pl}}},
$$

where $\beta_{\mathrm{BG}}=\beta\left(\phi_{\mathrm{BG}}\right)$. The thin shell condition is simply given by $\chi_{\mathrm{BG}}<|\Psi|$. This is called self-screening because this condition only takes into account the screening of the scalar field by an isolated object. The ratio of the scalar to Newtonian force outside a source acting on a non-relativistic particle is given by

$$
\frac{F_{\phi}}{F_{N}}=2 \beta_{\mathrm{BG}}^{2} \min \left\{\left(\frac{\chi_{\mathrm{BG}}}{|\Psi|}\right), 1\right\} .
$$

The above formula applies to the force acting on a test particle. The attraction due to the scalar field between two objects separated by distance $r$ is in general given by

$$
F_{1,2}=2 Q_{1} Q_{2} \frac{G M_{1} M_{2}}{r^{2}},
$$

where

$$
Q_{i}=\beta_{\mathrm{BG}} \min \left\{\left(\frac{\chi_{\mathrm{BG}}}{|\Psi|_{i}}\right), 1\right\},
$$

and $|\Psi|_{i}$ is the gravitational potential of the object $i$.

Table. $1^{63}$ summarises the gravitational potential of several classes of astrophysical objects, which are useful to estimate the self-screening of these objects.

\begin{tabular}{c|c} 
Object & Newtonian Potential $|\Psi|$ \\
\hline Earth & $10^{-9}$ \\
Moon & $10^{-11}$ \\
Main-sequence star (Sun-like) & $10^{-6}$ \\
Post-main-sequence star $\left(\mathcal{M}=1-10 M_{\odot}, R=10-100 R_{\odot}\right)$ & $10^{-7}-10^{-8}$ \\
Spiral galaxy (Milky Way-like, $\left.v_{c} \sim 200 \mathrm{~km} / \mathrm{s}\right)$ & $10^{-6}$ \\
Dwarf galaxy $\left(v_{c} \sim 50 \mathrm{~km} / \mathrm{s}\right)$ & $10^{-8}$
\end{tabular}

Table 1: Objects commonly considered as probes of chameleon/symmetron/dilaton screening. The second column shows surface Newtonian potential $|\Psi| \equiv G M / R$. Here $v_{c}$ is the circular velocity. From the reference. $[6]$

It was found that screening also depends on environments. For example, if an object with a deep potential exists nearby, this can screen an object that does not satisfy the self-screening condition. To identify the environmental screening, the full partial differential equation needs to be solved with a given distribution of matter. This was done in the context of N-body simulations. ${ }^{67]}$ It was found that 
environmental screening can be estimated by computing the gravitational potential of nearby objects within the Compton wavelength

$$
\Psi_{\mathrm{ext}}=\sum_{d_{i}<m\left(\phi_{\mathrm{BG}}\right)^{-1}+R_{i}} \frac{G M_{i}}{d_{i}},
$$

where $d_{i}$ is the distance to the neighbouring object with mass $M_{i}$ and radius $R_{i}$. The environmental screening condition is given by $\chi_{\mathrm{BG}}<\Psi_{\text {ext }}$. This condition was verified by studying screening of dark matter halos in N-body simulations and applied to SDSS galaxies within 200Mpc to create a screening map, which identifies the regions where environmental screening does not apply 68 A more sophisticated method has been developed to include the effect of missing mass and it was concluded that it is in principle possible to test the thin shell condition to the level of $\chi_{\mathrm{BG}} \sim 10^{-7} \underline{69}$

\subsubsection{Constraints}

There are various constraints on thin shell screened models.

(1) Cassini experiment

The constraint from the Casini experiment (12) imposes the condition that the modification of the unscreened Casini satellite in the vicinity of the Sun should be suppressed as

$$
\beta_{\text {galaxy }}^{2}\left(\frac{\chi_{\text {galaxy }}}{\left|\Psi_{\text {sun }}\right|}\right)<10^{-5}
$$

where the background is assumed to be determined by the Milky Way galaxy.

(2) Lunar Laser Ranging experiments

There is a strong constraint on the difference between the acceleration of the Earth and the Moon due to the Sun (15). If the Earth, Moon and Sun are thin-shell screened, the accelerations are given by

$$
a_{\text {earth }}=a_{N}\left(1+2 Q_{\text {earth }} Q_{\text {sun }}\right), \quad a_{\text {moon }}=a_{N}\left(1+2 Q_{\text {moon }} Q_{\text {sun }}\right) .
$$

Using the gravitation potential of the Earth, Moon and Sun in Table 1, the constraint can be written as

$$
\beta_{\text {galaxy }}^{2}\left(\frac{\chi_{\text {galaxy }}}{\left|\Psi_{\text {earth }}\right|}\right)^{2}<10^{-14}
$$

where again we assumed that the background is determined by the Milky Way galaxy.

(3) Milky Way galaxy constraint

The above constraints assume that the scalar field is at the minimum in the Milky Way galaxy. To ensure that the Milky Way galaxy is screened we need to impose the condition

$$
\beta_{\text {cosmology }}^{2}\left(\frac{\chi_{\text {cosmology }}}{\left|\Psi_{\text {galaxy }}\right|}\right)<1
$$


where the background is assumed to be determined by the cosmological background.

In addition, there are constraints from laboratory experiments and astrophysics tests,$\frac{63}{6}$ which will be discussed in details in the articles in this special issue.

Note that the Solar System constraints and the LLR constraint impose the condition on the scalar field with the galaxy density. To convert these constraints to those on model parameters, we need to specify the potential and coupling function. The galaxy density is given by $\rho_{\text {galaxy }} \sim 10^{5} \rho_{\text {cosmology }}$. The background scalar field scales as $\phi_{\mathrm{BG}} \propto \rho^{-1 /(\ell+1)}$ (see Eq. (41)). If $1 \ll 1 /(\ell+1)$, the condition for the Miky Way galaxy screening (66) typically gives the strongest constraint on $\phi_{\text {cosmology }}$ as $\phi_{\mathrm{BG}}$ is highly suppressed in denser environments ${ }^{70}$ This is the case in $f(R)$ gravity models discussed in section 5.1 where $2 \leq 1 /(\ell+1) ! 71$

\subsection{K-mouflage mechanism}

This mechanism can be described by the action

$$
S=\int d^{4} x \sqrt{-g}\left(\frac{1}{16 \pi G} R+\Lambda_{2}^{4} K\left(\frac{X}{\Lambda_{2}^{4}}\right)\right)+S_{m}\left[A(\phi)^{2} g_{\mu \nu}\right], \quad X=-\frac{1}{2} \partial^{\mu} \phi \partial_{\mu} \phi .
$$

The equation of motion for the scalar field is given by

$$
\frac{1}{\sqrt{-g}} \partial_{\mu}\left(\sqrt{-g} \partial^{\mu} \phi K^{\prime}\right)=\frac{d \ln A}{d \phi} \rho .
$$

We consider perturbations around a background $\phi=\phi_{\mathrm{BG}}+\varphi(x)$ and focus on quasi-static perturbations with a non-relativistic source. The equation of motion for $\varphi$ can be integrated once ${ }^{72}$

$$
K^{\prime} \nabla \varphi=2 \beta M_{\mathrm{pl}} \nabla \Psi,
$$

where the gravitational potential satisfies the normal Poisson equation

$$
\nabla^{2} \Psi=4 \pi G \delta \rho
$$

and

$$
K^{\prime} \equiv \frac{d K(\chi)}{d \chi}, \quad \chi=-\frac{1}{2 \Lambda_{2}^{4}}(\nabla \phi)^{2} .
$$

Note that in principle the energy density fluctuation $\delta \rho$ contains the energy density of the scalar field itself but this is sub-dominant compared with the matter density and we will ignore it. For a spherically symmetric object with mass $M$, we can define the K-mouflage distance as

$$
r_{K}=\left(\frac{2 \beta G M M_{\mathrm{pl}}}{\Lambda_{2}^{2}}\right)^{1 / 2} .
$$


For $r<r_{K},|\chi|$ becomes large and if $K^{\prime}(\chi)$ becomes large, the fifth force is suppressed:

$$
\frac{F_{\phi}}{F_{N}}=\frac{2 \beta^{2}}{K^{\prime}} .
$$

The condition for the screening $r<r_{K}$ can be written as

$$
\frac{G M}{r^{2}}>\Lambda_{c} \equiv \frac{\Lambda_{2}^{2}}{2 \beta M_{\mathrm{pl}}}
$$

Thus in this screening mechanism, screening is determined by the first derivative of the gravitational potential.

\subsubsection{Constraints}

The ratio of the scalar to Newtonian force is constrained by the Cassini experiment (12)

$$
\frac{F_{\phi}}{F_{N}}=\frac{2 \beta^{2}}{K^{\prime}}<10^{-5},
$$

at distances of order one AU from the Sun. Assuming $\Lambda_{2}$ is associated with dark energy energy scale, i.e. $\Lambda^{2} / M_{\mathrm{pl}} \sim H_{0}$ the K-mouflage radius $r_{K}$ is estimated as ${ }^{72}$

$$
r_{K} \sim 3500 \mathrm{AU} \sqrt{\frac{\beta M}{M_{\text {sun }}}} .
$$

The correction to the gravitational potential is the radial dependent and this introduces an anomalous perihelion for the Moon orbiting around the Earth given by 13 where the ratio between the scalar and Newtonian potential is given by

$$
\varepsilon=\frac{\beta \varphi}{M_{\mathrm{pl}} \Psi} \text {. }
$$

Using the equation of motion, this can be written as

$$
\delta \theta=-8 \pi \frac{\beta^{2}}{K^{\prime}} \frac{\chi K^{\prime \prime}}{K^{\prime}} \frac{1}{c_{s}^{2}}, \quad c_{s}^{2}=\frac{K^{\prime}+2 \chi K^{\prime \prime}}{K^{\prime}} .
$$

The Earth-Moon system gives a constraint $|\delta \theta|<2.4 \times 10^{-11}$.

\subsection{Vainshtein mechanism}

The Vainshtein mechanism is the oldest idea of the screening mechanism. This mechanism was identified in the context of massive gravity. Massive graviton has five degrees of freedom. In the linear theory, the helicity- 0 mode does not decouple even in the massless limit. This is known as the van Dam-Veltman-Zakharov discontinuity 73 T4 74 is problem is cured by non-linear interactions of the helicity-0 mode, which decouple this mode. This is known as the Vainshtein mechanism. The massive gravity models considered by Vainshtein contain a Boulware-Deser ghost ${ }^{75}$ but the same mechanism applies to a braneworld model ${ }^{30}$ and also a ghost-free massive gravity model ${ }^{[76}$ 


\subsubsection{Vainshtein mechanism}

The simplest model to describe the Vainshtein mechanism is given by the action

$$
\left.S=\int d^{4} x \sqrt{-g}\left(\frac{1}{16 \pi G} R-\frac{1}{2}(\partial \phi)^{2}-\frac{1}{2 \Lambda_{3}^{3}} \square \phi(\partial \phi)^{2}\right)+S_{m}\left[A(\phi)^{2} g_{\mu \nu}\right)\right] .
$$

We consider static perturbations around the Minkowski background for simplicity. The equation of motion for the scalar field is

$$
\nabla^{2} \phi+\frac{1}{\Lambda_{3}^{3}}\left[\left(\nabla^{2} \phi\right)^{2}-\left(\nabla_{i} \nabla_{j} \phi\right)\left(\nabla^{i} \nabla^{j} \phi\right)\right]=\frac{\beta \rho}{M_{\mathrm{pl}}} .
$$

Again we consider a spherically symmetric source and compute quasi-static perturbations. Outside the matter source, the solution is obtained

$$
\frac{1}{M_{\mathrm{pl}}} \frac{d \phi}{d r}=\frac{\beta G M}{r^{2}}\left(\frac{r}{r_{V}}\right)^{3}\left(\sqrt{1+4\left(\frac{r_{V}}{r}\right)^{3}}-1\right)
$$

where the Vainshtein radius is given by

$$
r_{V}=\left(\frac{4 \beta G M M_{\mathrm{pl}}}{\Lambda_{3}^{3}}\right)^{1 / 3}
$$

At large distance, we recover the linear solution. On the other hand, inside the Vainshtein radius $r \ll r_{V}$, we obtain

$$
\frac{1}{M_{\mathrm{pl}}} \frac{d \varphi}{d r}=\frac{2 \beta G M}{r^{2}}\left(\frac{r}{r_{V}}\right)^{3 / 2}
$$

and the ratio of the scalar to Newtonian force is given by

$$
\frac{F_{\phi}}{F_{N}}=2 \beta^{2}\left(\frac{r}{r_{V}}\right)^{3 / 2}
$$

The condition for the screening $r<r_{V}$ can be written as

$$
\frac{G M}{r^{3}}>\Lambda_{c}^{2} \equiv \frac{\Lambda_{3}^{3}}{4 \beta M_{\mathrm{pl}}} .
$$

The length scale associated with $\Lambda_{c}, r_{c} \equiv \Lambda_{c}^{-1}$, is typically assumed to be $r_{c} \sim H_{0}^{-1}$. Table 2 summarises the Vainshtein radius and the Schwarzchild radius $r_{\mathrm{g}}=2 G M$ for several objects with this choice of the parameter ${ }^{77}$

\subsubsection{Constraints}

The Vainshtein radius $r_{V}$ is much larger than the Schwarzschild radius for $r_{c} \sim H_{0}$. For the Sun, $r_{V}$ is significantly larger than the size of the Solar System. Thus inside the Solar System, the fifth fore contribution is well approximated by 83 . The ratio between the scalar and gravitation potential is given by

$$
\varepsilon=-4 \beta^{2}\left(\frac{r}{r_{V}}\right)^{3 / 2} \text {. }
$$




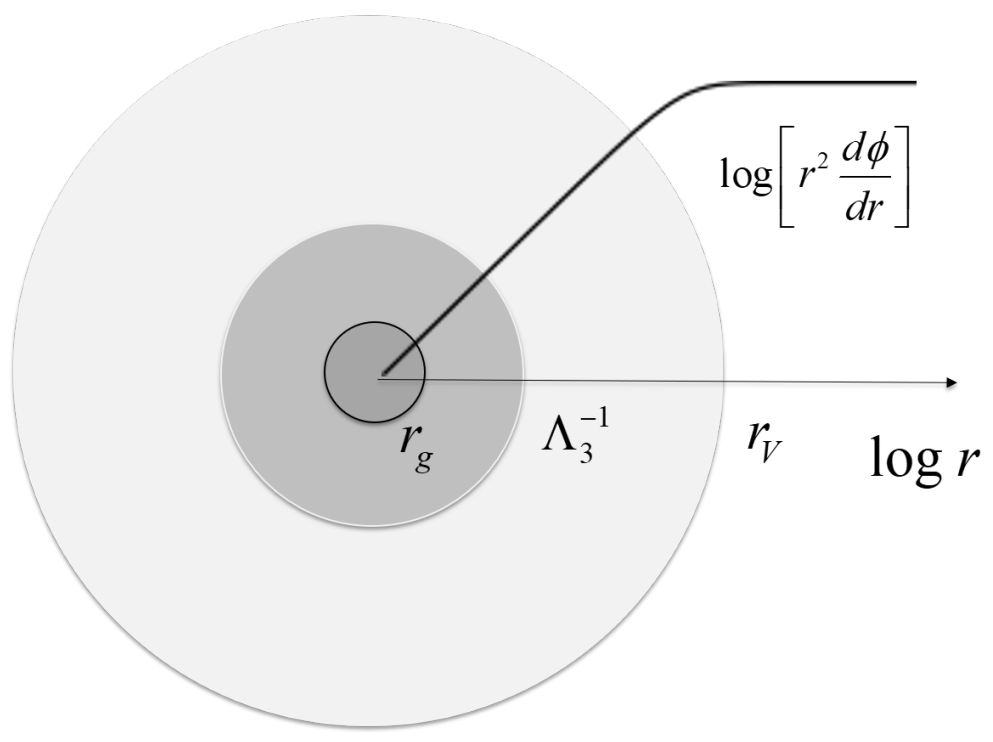

Fig. 4: The scalar field profile for a spherically symmetric solution with the Vainshtein mechanism. $r_{\mathrm{g}}=2 G M$ is the Schwarzchild radius.

\begin{tabular}{l|ccc} 
Object & \multicolumn{1}{|c}{$R$} & $r_{\mathrm{g}}$ & $r_{V}$ \\
\hline Milky Way & $\sim 0.9 \times 10^{21}$ & $\sim 2 \times 10^{15}$ & $\sim 3 \times 10^{22}$ \\
Sun & $\sim 0.7 \times 10^{9}$ & $\sim 3 \times 10^{3}$ & $\sim 3.5 \times 10^{18}$ \\
Earth & $\sim 6 \times 10^{6}$ & $\sim 9 \times 10^{-3}$ & $\sim 5 \times 10^{16}$ \\
Atom & $\sim 5 \times 10^{-11}$ & $\sim 1.8 \times 10^{-54}$ & $\sim 3 \times 10^{-1}$
\end{tabular}

Table 2: The radii $R$ (physical size), $r_{\mathrm{g}}=2 G M$ (Schwarzschild radius) and $r_{V}$ (Vainshtein radius) of some typical objects. Unit is meter. For estimation we have used $\beta=2 \sqrt{2} / 3$ and $r_{\mathrm{c}}=H_{0}^{-1} \sim 4000 \mathrm{Mpc} \sim 1.2 \times 10^{26} \mathrm{~m}$.

Note that the scalar field is normalised so that it vanishes at infinity. The perihelion precession per orbit 13 is then calculated as

$$
\delta \theta=\frac{3}{4} \pi \varepsilon .
$$

Lunar Laser Ranging experiments put constraints $|\delta \theta|<2.4 \times 10^{-11}$. Using $r_{g}=$ $2 G M=0.886 \mathrm{~cm}$ for the Earth and $r=3.84 \times 10^{10} \mathrm{~cm}$ for the Earth-Moon distance we obtain $r_{c}>350 \mathrm{Mpc}$ for $\beta \sim O(1){ }^{79}$ These results are obtained by treating the Moon as a test body. This assumption is not necessarily valid. For example for the Earth-Moon system, it was shown that there is a correction to the universal precession rate (87) due to the nonsuperimposability of the field that depends on the mass ratio of the two bodies although the effect is small (4\%) for the Earth-Moon system. ${ }^{80}$ See the reference ${ }^{81}$ for the constraints from laboratory tests. 


\section{Examples of modified gravity models}

In this section, we will review several modified gravity models focusing on those that will be used in other articles in the special issue.

\section{1. $f(R)$ gravity}

One of the most popular modified gravity models is an $f(R)$ gravity where the Einstein-Hilbert action is generalised to be a function of the Ricci curvature scalar ${ }^{82}$

$$
S=\frac{1}{16 \pi G} \int d^{4} x \sqrt{-g} F(R)+\int d^{4} x \sqrt{-g} \mathcal{L}_{m} .
$$

See the references ${ }^{\sqrt{83}} \sqrt{85}$ for reviews. The modified Einstein equations are given by

$$
F_{R} R_{\mu \nu}-\frac{1}{2} g_{\mu \nu} F(R)+\left(g_{\mu \nu} \square-\nabla_{\mu} \nabla_{\mu} \nu\right) F_{R}=8 \pi G T_{\mu \nu} .
$$

These equations contain up to fourth order derivatives in terms of $g_{\mu \nu}$. However, this theory is an example where the non-degeneracy condition for the Ostrogradsky theorem is not satisfied. Indeed, it is possible to introduce a scalar field and make the equation of motion second order. The action is equivalent to

$$
S=\frac{1}{16 \pi G} \int d^{4} x \sqrt{-g}\left(F(\phi)+(R-\phi) F^{\prime}(\phi)\right) .
$$

By taking a variation with respect to $\phi$, we obtain $(R-\phi) F^{\prime \prime}(\phi)=0$. As long as $F^{\prime \prime}(\phi) \neq 0, R=\phi$ and we recover the original action. By defining $\psi=F^{\prime}(\phi)$ and $2 U=-F(\phi)+\phi F^{\prime}(\phi)$, the action can be written as the same form as Brans-Dicke gravity with a potential

$$
S=\frac{1}{16 \pi G} \int d^{4} x \sqrt{-g}(\psi R-2 U(\psi)) .
$$

Comparing this with the action $(20)$, we notice that the $\mathrm{BD}$ parameter is given by $\omega_{\mathrm{BD}}=0$. As such, if we ignore the potential, this model is already excluded by the Solar System constraints. However, by choosing the potential, i.e. the form of the $F(R)$ function appropriately, it is possible to incorporate a chameleon mechanism to evade the Solar System constraint 7186 It is often more convenient to extract the Einstein-Hilbert action in $F(R)$ so we define

$$
F(R)=R+f(R) .
$$

A choice of the function $f(R)$ leads to many models but the successful models for the late time cosmology share the same features $\frac{71] 87}{88}$ In the high curvature limit, the function looks like

$$
f(R)=-2 \Lambda+\left|f_{R 0}\right| \frac{\bar{R}_{0}^{n+1}}{R^{n}},
$$

where $\bar{R}_{0}$ is the background curvature today. This model requires an effective cosmological constant to explain the observed accelerated expansion of the universe. 
The correction to $\Lambda$ CDM disappears in the high curvature limit $R \gg \bar{R}$. This suggests the existence of a screening mechanism in this model. In the Einstein frame, the potential and the coupling function take the following form

$$
V(\phi)=V_{0}-\Lambda^{\frac{3 n+4}{1+n}} \phi^{\frac{n}{1+n}}, \quad A(\phi)^{2}=e^{\sqrt{2 / 3} \phi / M_{\mathrm{Pl}}},
$$

and the scalar field is related to $f_{R} \equiv d f / d R$ as

$$
\phi=-\sqrt{\frac{3}{2}} M_{\mathrm{Pl}} \log \left(1+f_{R}\right) .
$$

This is a particular case of the chameleon theories where the coupling constant is given by $\beta=d \ln A / d \phi=\sqrt{1 / 6}$ and the potential given by 40 with $\ell=-n /(1+n)$.

Under quasi-static approximations, the modified Poisson equation and the perturbations of $f_{R}$ is given by ${ }^{71}$

$$
\begin{aligned}
\nabla^{2} \delta f_{R} & =\frac{a^{2}}{3}\left[\delta R\left(f_{R}\right)-8 \pi G \delta \rho\right], \\
\nabla^{2} \Psi & =\frac{16 \pi G}{3} a^{2} \delta \rho-\frac{a^{2}}{6} \delta R\left(f_{R}\right),
\end{aligned}
$$

where $\delta f_{R}=f_{R}(R)-f_{R}(\bar{R}), \delta R=R-\bar{R}$ and $\delta \rho=\rho-\bar{\rho}$. If we linearise the equation, $\delta R\left(f_{R}\right)$ is given by

$$
\delta R\left(f_{R}\right)=3 m^{2} \delta f_{R}, \quad m^{2}=\frac{1}{3 f_{R R}} .
$$

Note that in order to avoid tachyonic instabilities, we need to impose $f_{R R}>0$. The solution for the gravitational potential is given by 89

$$
k^{2} \Psi=-4 \pi G\left(\frac{4+3 a^{2} m^{2} / k^{2}}{3+3 a^{2} m^{2} / k^{2}}\right) a^{2} \delta \rho,
$$

where we performed the Fourie transformation and $k$ is the comoving wavenumber. On scales larger than the Compton wavelength $k / a<m^{-1}$, the scalar field does not propagate and we recover the standard solution. On the other hand, on scales smaller than the Compton wavelength $k / a>m^{-1}$, gravity is enhanced by the fifth force and the effective Newton constant becomes $4 G / 3$ as expected from the fact that $f(R)$ gravity has $\omega_{\mathrm{BD}}=0$ (see $(26)$ ). For a model with $n=1$, the mass is given by the background Ricci curvature as

$$
m^{2}=\frac{1}{6\left|f_{R 0}\right|} \frac{\bar{R}^{3}}{\bar{R}_{0}^{2}} .
$$

At early times, the background Ricci curvature is large and the mass is large. Thus deviations from $\Lambda \mathrm{CDM}$ is suppressed. At late times, the Compton wavelength becomes large, modifying gravity on cosmological and astrophysical scales. The compton wavelength today is given by

$$
m_{0}^{-1}=32 \sqrt{\frac{\left|f_{R 0}\right|}{10^{-4}}} \mathrm{Mpc} .
$$


For non-linear structures, it is not possible to linearise the equation and we need to solve fully non-linear equations. This requires significant efforts in particular in the context of N-body simulations. This will be one of the main topics of this special issue. Note that the self-screening condition is given in this model by

$$
\chi_{\mathrm{BG}}=\frac{3}{2}\left|f_{R}(\bar{R})\right|<|\Psi| .
$$

The constraint that the Milky Way galaxy is screened 66 imposes the condition $\left|f_{R 0}\right|<10^{-6}$. This constraint implies that $m_{0}^{-1}<3 \mathrm{Mpc}$. Thus the scalar field dynamics is not important cosmologically and the background expansion histroy is observationally indistinguishable from that of $\Lambda \mathrm{CDM}^{[86}$ This is a general conclusion in models with the chameleon mechanism.90

For a general choice of $f(R)$, it is possible to mimic the $\Lambda$ CDM expansion history or any expansion history by tuning the function $f(R)$ provided that $R$ does not have a minimum in the past (see Ref. ${ }^{91}$ for discussions). This is called designer models ${ }^{92}$ In the designer models, linear cosmological perturbations are characterised by the Compton wavelength parameter

$$
B(a)=\frac{f_{R R}(\bar{R})}{1+f_{R}(\bar{R})} \bar{R}^{\prime} \frac{H}{H^{\prime}},
$$

where $\bar{R}$ is the background Ricci curvature, $H$ is the Hubble parameter and the prime denotes the derivative with respect to the scale factor.

Finally, the speed of gravitational waves is the same as that of light in $f(R)$ gravity models thus the constraint 17) does not impose any condition on the model.

\subsection{Braneworld gravity}

The idea of braneworld was inspired by the discovery of D-branes in string theory and subsequent developments of large extra-dimensions ( $\sec ^{27}$ for a review). In this model, we are living on a four-dimensional membrane (brane) in a higher dimensional spacetime (bulk). The standard model particles are confined to the brane but gravity can propagate in the bulk. In these models, it is possible to have a situation where higher dimensional gravity becomes important on large scales. The simplest model is the Dvali-Gabadadze-Porrati (DGP) model described by the action 130

$$
S=\frac{1}{16 \pi G_{(5)}} \int d^{5} x \sqrt{-^{(5)} g^{(5)}} R+\int d^{4} x \sqrt{-g}\left(\frac{1}{16 \pi G} R+\mathcal{L}_{m}\right),
$$

where ${ }^{(5)} R$ and $G_{(5)}$ are five-dimensional Ricci curvature and Newton constant, respectively. The ratio between the five-dimensional and four-dimensional Newton constant $r_{c}=G_{(5)} / 2 G$ is a parameter of the model and called the cross-over radius. The Friedman equation on the brane is given by

$$
H^{2}= \pm \frac{H}{r_{c}}+\frac{8 \pi G}{3} \rho \text {. }
$$


At early times $H r_{c} \gg 1$, we recover the usual 4D Friedmann equation. On the other hand, at late times, the Hubble parameter approaches a constant $H \rightarrow 1 / r_{c}$ in the upper branch of the solution. This is known as the self-accelerating branch. On the other hand, the lower branch solution, the normal branch solution, requires the cosmological constant or dark energy to realise the accelerated expansion of the Universe. In order to recover the standard cosmology, the cross-over radius needs to be tuned as $r_{c} \sim H_{0}^{-1}$.

The perturbations around this background under the quasi-static approximations are described by the Brand Dicke theory with an additional non-linear interaction term $78|94| 95$

$$
\begin{gathered}
\nabla^{2} \Psi=4 \pi G a^{2} \rho-\frac{1}{2} \nabla^{2} \varphi, \\
\left(3+2 \omega_{\mathrm{BD}}(a)\right) \nabla^{2} \varphi+\frac{r_{c}^{2}}{a^{2}}\left[\left(\nabla^{2} \varphi\right)^{2}-\left(\nabla_{i} \nabla_{j} \varphi\right)^{2}\right]=-8 \pi G a^{2} \rho, \\
\Phi-\Psi=\varphi,
\end{gathered}
$$

where the $\mathrm{BD}$ parameter $\omega_{B D}(a)$ is given by

$$
\omega_{\mathrm{BD}}=\frac{3}{2}(\beta(a)-1), \quad \beta(a)=1 \mp 2 r_{c} H\left(1+\frac{\dot{H}}{3 H^{2}}\right),
$$

where dot indicates a derivative with respect to time.

Note that the Brans Dicke parameter is always negative in the self-accelerated branch and the scalar field mediates a repulsive force. This is the manifestation of the problem that the self-accelerating solutions suffer from a ghost instability 31$] 32[96] 98$ In addition, at late times $\omega_{\mathrm{BD}} \sim O(1)$ thus by linearising the equation, we again find that this theory would be excluded by the Solar System constraints. However, the coefficient of the non-linear interaction term is very large, $r_{c}^{2} \sim H_{0}^{-2}$. This is responsible for the Vainshtein mechanism. ${ }^{99}$ Indeed, the equation (107) has the same form as 80 so the constraint from the LLR imples $r_{c}>$ a few hundreds Mpc.

In the normal branch solution, it is possible to tune the equation of state of dark energy to realise the $\Lambda \mathrm{CDM}$ background expansion history 100 This is not particularly well-motivated theoretically. However, this model makes it easier to study the effect of the Vainshtein mechanism on structure formation compared with $\Lambda \mathrm{CDM}$ and thus it is frequently used in N-body simulations and phenomenological studies.

On large scales $r>r_{c}$, gravitational waves propagate in the bulk while light is confined to the brane. Thus there can be a time delay between the arrival of gravitational waves and light as gravitational waves can make a short-cut in the bulk ${ }^{101}$ However, the event GW170817 happens at the distance $\sim 40 \mathrm{Mpc}$ from us and the frequencies of the gravitational waves are $10-100 \mathrm{~Hz}$. Due to the shortwavelength of the gravitational waves $\left(10^{-16}-10^{-15} \mathrm{Mpc}\right)$, the time delay will be well suppressed. For example, for a single massive graviton, the constraint (17) gives only a weak constraint on the graviton mass $m<10^{-22} \mathrm{eV}\left(m^{-1}>10^{-7} \mathrm{Mpc}\right)$. 
Thus once we impose the LLR constraint, the constraint (17) is well satisfied in this model.

\subsection{Horndeski theory and beyond}

Scalar-tensor theories of gravity represent the simplest modification in terms of additional degrees of freedom compared to general relativity, i.e. a single scalar field. In four-dimensional spacetime, the most general scalar-tensor theory with second order equations of motion was derived by Horndeski in 1974, 102 and later rediscovered in the context of the so-called (covariant) galileon theories $\frac{103}{108}$ See the reference ${ }^{109}$ for a review. The Horndeski action is given by

$$
\begin{aligned}
\mathcal{L}_{2} & =K(\phi, X), \\
\mathcal{L}_{3} & =G_{3}(\phi, X) \square \phi, \\
\mathcal{L}_{4} & =G_{4}(\phi, X) R-2 G_{4 X}(\phi, X)\left[(\square \phi)^{2}-\left(\nabla_{\mu} \nabla_{\nu} \phi\right)^{2}\right], \\
\mathcal{L}_{5} & =G_{5}(\phi, X) G^{\mu \nu} \nabla_{\mu} \nabla_{\nu} \phi \\
& +\frac{1}{3} G_{5 X}(\phi, X)\left[(\square \phi)^{3}-3 \square \phi\left(\nabla_{\mu} \nabla_{\nu} \phi\right)^{2}+2\left(\nabla_{\mu} \nabla_{\nu} \phi\right)^{3}\right],
\end{aligned}
$$

where $X=\partial^{\mu} \phi \partial_{\mu} \phi$ and $K, G_{3}, G_{4}$ and $G_{5}$ are free function of the scalar field and $X$.

This theory encompasses many models including $f(R)$ gravity and screened models discussed above. These theories have $G_{4}=G_{4}(\phi)$ and $G_{5}=0$. The extension of these theories to non-trivial $G_{4}(\phi, X)$ and $G_{5}(\phi, X)$ has a significant implication for the propagation of gravitational waves on the cosmological background. The propagation of gravitational waves

$$
d s^{2}=-d t^{2}+a^{2}\left(\delta_{i j}+h_{i j}\right) d x^{i} d x^{j}, \quad h_{i}^{i}=\partial_{i} h_{j}^{i}=0,
$$

is described by the following equation 110

$$
\ddot{h}_{i j}+\left(3+\alpha_{M}\right) H \dot{h}_{i j}+c_{\mathrm{GW}}^{2} \frac{\nabla^{2}}{a^{2}} h_{i j}=0,
$$

where the tensor sound speed is given by

$$
c_{\mathrm{GW}}^{2}=1+\alpha_{T}
$$

and

$$
\begin{aligned}
M_{*}^{2} & =2\left[G_{4}-2 X G_{4 X}-\frac{1}{2} X G_{5 \phi}-\dot{\phi} H X G_{5 X}\right], \\
H M_{*}^{2} \alpha_{M} & =\frac{d M_{*}^{2}}{d t}, \\
M_{*}^{2} \alpha_{T} & =2 X\left[2 G_{4 X}+G_{5 \phi}-(\ddot{\phi}-H \dot{\phi}) G_{5 X}\right] .
\end{aligned}
$$

The constraint on the speed of gravitational waves (17) gives a strong constraint $\left|\alpha_{T}\right|<10^{-15}$. If we do not invoke a fine tuning and set $c_{\mathrm{GW}}=c$, this forces us to choose

$$
G_{4}=G_{4}(\phi), \quad G_{5}=0 .
$$


The screening mechanisms introduced earlier, i.e. chameleon, symmetron and dilaton models, satisfy these conditions so they are not affected by this constraint. Note also that $f(R)$ gravity is also included in this class of models.

A theory with non-zero $K(\phi, X)$ and $G_{3}(\phi, X)$ are called Kinetic Gravity Brading (KGB) model 1111 One interesting feature of this model is that it is possible to explain the accelerated expansion of the Universe without introducing a potential. A simple example is given by $\underline{112}$

$$
K(X)=\frac{X}{2}, \quad G_{3}(X)=-M_{\mathrm{Pl}}\left(-\frac{r_{c}^{2}}{2 M_{\mathrm{Pl}}^{2}} X\right)^{n} .
$$

Note that the sign for the kinetic term is opposite to the normal scalar field. However, this does not imply that the perturbations are ghostly when expanding around the non-trivial cosmological background. The strong observational constraints in this model come from the Integrated Sachs-Wolfe (ISW) effects. The cross-correlation between ISW and galaxy clustering has a different sign compared with the $\Lambda$ CDM model for samll $n$, which is disfavoured. This gives a condition on $n$ to be larger than a few hundreds $113 \mid 114$

The requirement of second order equations of motion is sufficient to avoid the Ostrogradsky ghost, but this condition is not necessary 115$] 115-118$ In recent years, there have been several attempts to construct healthy theories that relax this condition, exploiting transformations of the metric $119 \mid 120$ This brought initially to the class of beyond Horndeski theories 120121 A breakthrough in the subject came with the works of Refs. $\frac{48[122]}{1}$ which developed a general method to identify the degeneracy conditions that remove the Ostrogradsky ghost despite the appearance of higher derivatives in the equation of motion. A larger class of new degenerate higher order scalar-tensor theories propagating up to 3 degrees of freedom was identified and classified up to cubic order in the second order derivative of the scalar field! 123

Again the constraint on $c_{\mathrm{GW}}$ has significant implications to this extended class of scalar tensor theories. If we impose $c_{\mathrm{GW}}=c$, we only left with the following possibility in addition to $K(\phi, X)$ and $G_{3}(\phi, X) ! \frac{126}{1.126}$

$$
\mathcal{L}_{4}=\sum_{i=1}^{5} \mathcal{L}_{i}+\mathcal{L}_{R}
$$

where

$$
\begin{aligned}
& \mathcal{L}_{1}\left[A_{1}\right]=A_{1}(\phi, X) \phi_{\mu \nu} \phi^{\mu \nu}, \mathcal{L}_{2}\left[A_{2}\right]=A_{2}(\phi, X)(\square \phi)^{2}, \\
& \mathcal{L}_{3}\left[A_{3}\right]=A_{3}(\phi, X)(\square \phi) \phi^{\mu} \phi_{\mu \nu} \phi^{\nu}, \mathcal{L}_{4}\left[A_{4}\right]=A_{4}(\phi, X) \phi^{\mu} \phi_{\mu \rho} \phi^{\rho \nu} \phi_{\nu}, \\
& \mathcal{L}_{5}\left[A_{5}\right]=A_{5}(\phi, X)\left(\phi^{\mu} \phi_{\mu \nu} \phi^{\nu}\right)^{2}, \mathcal{L}_{R}\left[G_{4}\right]=G_{4}(\phi, X) R,
\end{aligned}
$$


and the functions $A_{I}$ satisfy

$$
\begin{aligned}
& A_{1}=0 \\
& A_{2}=0, \quad A_{5}=\frac{A_{3}}{2 G_{4}}\left(4 G_{4 X}+A_{3} X\right), \\
& A_{4}=-\frac{1}{8 G}\left[8 A_{3} G_{4}-48 G_{4 X}^{2}-8 A_{3} G_{4 X} X+A_{3}^{2} X^{2}\right],
\end{aligned}
$$

This theory has an interesting implication for the Vainshtein mechanism. We introduce a mass dimension $\Lambda_{3}$ and assume the following scaling for the functions $G_{4}, A_{3}, A_{4}$ and $A_{5}$

$$
G_{4} \sim M_{p}^{2}, X A_{3} \sim X A_{4} \sim X^{2} A_{5} \sim M_{p} \Lambda^{-3},
$$

where $M_{\mathrm{pl}}$ is the Planck mass and $X \sim M_{\mathrm{pl}} \Lambda_{3}^{3}$. There appears the Vainshtein radius $r_{V}=\left(M / M_{\mathrm{pl}} \Lambda^{3}\right)^{1 / 3}$ below which the scalar field perturbation becomes non-linear for a spherically symmetric object with mass $M$. The spherically symmetric solution for the metric perturbations below the Vainshtein radius is given by 127$] 129$

$$
\begin{aligned}
\Psi^{\prime} & =\frac{G_{N} M}{r^{2}}+\frac{\Upsilon_{1} G_{N}}{4} M^{\prime \prime}, \\
\Phi^{\prime} & =\frac{G_{N} M}{r^{2}}-\frac{5 \Upsilon_{2} G_{N}}{4 r} M^{\prime}+\Upsilon_{3} G_{N} M^{\prime \prime},
\end{aligned}
$$

where

$$
\begin{gathered}
\Upsilon_{1}=-\frac{\left(4 G_{4 X}-X A_{3}\right)^{2}}{4 A_{3} G_{4}}, \\
\Upsilon_{2}=\frac{8 G_{4 X} X}{5 G_{4}}, \\
\Upsilon_{3}=-\frac{-16 G_{4 X}^{2}+A_{3}^{2} X^{2}}{16 A_{3} G_{4}}, \\
G_{N}=\left[8 \pi\left(2 G_{4}-2 X G_{4 X}-3 A_{3} X^{2} / 2\right)\right]^{-1} .
\end{gathered}
$$

Outside the matter source, $M$ is constant and we recover general relativity solutions inside the Vainshtein radius. On the other hand, inside a source, gravity is modified so the Vainshtein mechanism is broken. This gives an interesting possibility to test these theories using astrophysical objects. ${ }^{[130}$ Note that there is a strong constraint from the Hulse-Taylor binary pulsars. The gravitational constant for tensor perturbations $G_{\mathrm{GW}}$ is given by $G_{4}$, which is different from $G_{N}$. This changes the prediction of the orbital decay ${ }^{1311}$ From the constraint $(16)$, we obtain the constraint ${ }^{132}$

$$
-7.5 \times 10^{-3}<\frac{G_{\mathrm{GW}}}{G_{N}}-1<2.5 \times 10^{-3},
$$

where

$$
\frac{G_{\mathrm{GW}}}{G_{N}}-1=\frac{2 X G_{4 X}}{G_{4}}+\frac{3 A_{3} X^{2}}{2 G_{4}} .
$$


The modification to the gravitational potential $\Psi$ is constrained as

$$
-2 / 3<\Upsilon_{1}<1.6
$$

from the stellar structure ${ }^{133-135}$

In addition, these theories admit the self-accelerating solutions where the accelerated expansion can be realised without introducing the cosmological constant or the potential of the scalar field 136

\section{Conclusion}

This article gave an introduction to theories of modified gravity with a particular focus on screening mechanisms. Several examples of modified gravity models were discussed that accommodate the screening mechanisms. These models can evade the stringent constraints from the Solar System tests thanks to the screening mechanism. In addition, they avoid the stringent constraint on the gravitational wave speed obtained from the almost simultaneous detections of gravitational waves and short gamma ray bursts made on 17 August 2017. Thus these models serve as a test bed to constrain deviations from general relativity using laboratory experiments, astrophysical objects and cosmological observations. The other articles in this special issue will discuss methodologies to perform these tests, show the latest results from these novel tests of general relativity and discuss future prospects.

\section{Acknowledgement}

KK is supported by the STFC grant ST/N000668/1. The work of KK has received funding from the European Research Council (ERC) under the European Union's Horizon 2020 research and innovation programme (grant agreement 646702 "CosTesGrav").

\section{References}

1. Planck Collaboration, P. A. R. Ade, N. Aghanim, M. Arnaud, M. Ashdown, J. Aumont, C. Baccigalupi, A. J. Banday, R. B. Barreiro, J. G. Bartlett and et al., $A \mathscr{E} A$ 594 (September 2016) A13, arXiv:1502.01589

2. B. P. Abbott, R. Abbott, T. D. Abbott, F. Acernese, K. Ackley, C. Adams, T. Adams, P. Addesso, R. X. Adhikari, V. B. Adya and et al., ApJL 848 (October 2017) L13, arXiv:1710.05834 [astro-ph.HE].

3. Planck Collaboration, P. A. R. Ade, N. Aghanim, C. Armitage-Caplan, M. Arnaud, M. Ashdown, F. Atrio-Barandela, J. Aumont, C. Baccigalupi, A. J. Banday and et al., A\&A 571 (November 2014) A16, arXiv:1303.5076

4. A. G. Riess, A. V. Filippenko, P. Challis, A. Clocchiatti, A. Diercks, P. M. Garnavich, R. L. Gilliland, C. J. Hogan, S. Jha, R. P. Kirshner, B. Leibundgut, M. M. Phillips, D. Reiss, B. P. Schmidt, R. A. Schommer, R. C. Smith, J. Spyromilio, C. Stubbs, N. B. Suntzeff and J. Tonry, AJ 116 (September 1998) 1009, astro-ph/9805201.

5. S. Perlmutter, G. Aldering, G. Goldhaber, R. A. Knop, P. Nugent, P. G. Castro, S. Deustua, S. Fabbro, A. Goobar, D. E. Groom, I. M. Hook, A. G. Kim, M. Y. Kim, 
J. C. Lee, N. J. Nunes, R. Pain, C. R. Pennypacker, R. Quimby, C. Lidman, R. S. Ellis, M. Irwin, R. G. McMahon, P. Ruiz-Lapuente, N. Walton, B. Schaefer, B. J. Boyle, A. V. Filippenko, T. Matheson, A. S. Fruchter, N. Panagia, H. J. M. Newberg, W. J. Couch and T. S. C. Project, ApJ 517 (June 1999) 565, astro-ph/9812133.

6. D. J. Eisenstein, I. Zehavi, D. W. Hogg, R. Scoccimarro, M. R. Blanton, R. C. Nichol, R. Scranton, H.-J. Seo, M. Tegmark, Z. Zheng, S. F. Anderson, J. Annis, N. Bahcall, J. Brinkmann, S. Burles, F. J. Castander, A. Connolly, I. Csabai, M. Doi, M. Fukugita, J. A. Frieman, K. Glazebrook, J. E. Gunn, J. S. Hendry, G. Hennessy, Z. Ivezić, S. Kent, G. R. Knapp, H. Lin, Y.-S. Loh, R. H. Lupton, B. Margon, T. A. McKay, A. Meiksin, J. A. Munn, A. Pope, M. W. Richmond, D. Schlegel, D. P. Schneider, K. Shimasaku, C. Stoughton, M. A. Strauss, M. SubbaRao, A. S. Szalay, I. Szapudi, D. L. Tucker, B. Yanny and D. G. York, ApJ 633 (November 2005) 560, astro-ph/0501171.

7. W. J. Percival, S. Cole, D. J. Eisenstein, R. C. Nichol, J. A. Peacock, A. C. Pope and A. S. Szalay, MNRAS 381 (November 2007) 1053, arXiv:0705.3323.

8. Planck Collaboration, P. A. R. Ade, N. Aghanim, M. Arnaud, M. Ashdown, J. Aumont, C. Baccigalupi, A. J. Banday, R. B. Barreiro, N. Bartolo and et al., A $\& A 594$ (September 2016) A14, arXiv:1502.01590

9. H. Hildebrandt, M. Viola, C. Heymans, S. Joudaki, K. Kuijken, C. Blake, T. Erben, B. Joachimi, D. Klaes, L. Miller, C. B. Morrison, R. Nakajima, G. Verdoes Kleijn, A. Amon, A. Choi, G. Covone, J. T. A. de Jong, A. Dvornik, I. Fenech Conti, A. Grado, J. Harnois-Déraps, R. Herbonnet, H. Hoekstra, F. Köhlinger, J. McFarland, A. Mead, J. Merten, N. Napolitano, J. A. Peacock, M. Radovich, P. Schneider, P. Simon, E. A. Valentijn, J. L. van den Busch, E. van Uitert and L. Van Waerbeke, MNRAS 465 (February 2017) 1454, arXiv:1606.05338

10. DES Collaboration, T. M. C. Abbott, F. B. Abdalla, A. Alarcon, J. Aleksić, S. Allam, S. Allen, A. Amara, J. Annis, J. Asorey, S. Avila, D. Bacon, E. Balbinot, M. Banerji, N. Banik, W. Barkhouse, M. Baumer, E. Baxter, K. Bechtol, M. R. Becker, A. BenoitLévy, B. A. Benson, G. M. Bernstein, E. Bertin, J. Blazek, S. L. Bridle, D. Brooks, D. Brout, E. Buckley-Geer, D. L. Burke, M. T. Busha, D. Capozzi, A. Carnero Rosell, M. Carrasco Kind, J. Carretero, F. J. Castander, R. Cawthon, C. Chang, N. Chen, M. Childress, A. Choi, C. Conselice, R. Crittenden, M. Crocce, C. E. Cunha, C. B. D'Andrea, L. N. da Costa, R. Das, T. M. Davis, C. Davis, J. De Vicente, D. L. DePoy, J. DeRose, S. Desai, H. T. Diehl, J. P. Dietrich, S. Dodelson, P. Doel, A. Drlica-Wagner, T. F. Eifler, A. E. Elliott, F. Elsner, J. Elvin-Poole, J. Estrada, A. E. Evrard, Y. Fang, E. Fernandez, A. Ferté, D. A. Finley, B. Flaugher, P. Fosalba, O. Friedrich, J. Frieman, J. García-Bellido, M. Garcia-Fernandez, M. Gatti, E. Gaztanaga, D. W. Gerdes, T. Giannantonio, M. S. S. Gill, K. Glazebrook, D. A. Goldstein, D. Gruen, R. A. Gruendl, J. Gschwend, G. Gutierrez, S. Hamilton, W. G. Hartley, S. R. Hinton, K. Honscheid, B. Hoyle, D. Huterer, B. Jain, D. J. James, M. Jarvis, T. Jeltema, M. D. Johnson, M. W. G. Johnson, T. Kacprzak, S. Kent, A. G. Kim, A. King, D. Kirk, N. Kokron, A. Kovacs, E. Krause, C. Krawiec, A. Kremin, K. Kuehn, S. Kuhlmann, N. Kuropatkin, F. Lacasa, O. Lahav, T. S. Li, A. R. Liddle, C. Lidman, M. Lima, H. Lin, N. MacCrann, M. A. G. Maia, M. Makler, M. Manera, M. March, J. L. Marshall, P. Martini, R. G. McMahon, P. Melchior, F. Menanteau, R. Miquel, V. Miranda, D. Mudd, J. Muir, A. Möller, E. Neilsen, R. C. Nichol, B. Nord, P. Nugent, R. L. C. Ogando, A. Palmese, J. Peacock, H. V. Peiris, J. Peoples, W. J. Percival, D. Petravick, A. A. Plazas, A. Porredon, J. Prat, A. Pujol, M. M. Rau, A. Refregier, P. M. Ricker, N. Roe, R. P. Rollins, A. K. Romer, A. Roodman, R. Rosenfeld, A. J. Ross, E. Rozo, E. S. Rykoff, M. Sako, A. I. Salvador, S. Samuroff, 
C. Sánchez, E. Sanchez, B. Santiago, V. Scarpine, R. Schindler, D. Scolnic, L. F. Secco, S. Serrano, I. Sevilla-Noarbe, E. Sheldon, R. C. Smith, M. Smith, J. Smith, M. Soares-Santos, F. Sobreira, E. Suchyta, G. Tarle, D. Thomas, M. A. Troxel, D. L. Tucker, B. E. Tucker, S. A. Uddin, T. N. Varga, P. Vielzeuf, V. Vikram, A. K. Vivas, A. R. Walker, M. Wang, R. H. Wechsler, J. Weller, W. Wester, R. C. Wolf, B. Yanny, F. Yuan, A. Zenteno, B. Zhang, Y. Zhang and J. Zuntz, ArXiv e-prints (August 2017) arXiv:1708.01530.

11. A. G. Riess, S. Casertano, W. Yuan, L. Macri, J. Anderson, J. W. Mackenty, J. B. Bowers, K. I. Clubb, A. V. Filippenko, D. O. Jones and B. E. Tucker, ArXiv e-prints (January 2018) arXiv:1801.01120 [astro-ph.SR]

12. B. P. Abbott, R. Abbott, T. D. Abbott, F. Acernese, K. Ackley, C. Adams, T. Adams, P. Addesso, R. X. Adhikari, V. B. Adya and et al., Nature 551 (November 2017) 85, arXiv:1710.05835.

13. S. Weinberg, Reviews of Modern Physics 61 (January 1989) 1.

14. S. R. Green and R. M. Wald, Classical and Quantum Gravity 31 (December 2014) 234003, arXiv:1407.8084 [gr-qc].

15. T. Buchert, M. Carfora, G. F. R. Ellis, E. W. Kolb, M. A. H. MacCallum, J. J. Ostrowski, S. Räsänen, B. F. Roukema, L. Andersson, A. A. Coley and D. L. Wiltshire, Classical and Quantum Gravity 32 (November 2015) 215021, arXiv:1505.07800 [gr-qc].

16. K. Tomita, ArXiv e-prints (June 2009) arXiv:0906.1325 [astro-ph.CO]

17. T. Clifton, P. G. Ferreira, A. Padilla and C. Skordis, PhR 513 (March 2012) 1, arXiv:1106.2476,

18. A. Joyce, B. Jain, J. Khoury and M. Trodden, PhR 568 (March 2015) 1, arXiv:1407.0059.

19. K. Koyama, Reports on Progress in Physics 79 (April 2016) 046902, arXiv:1504.04623.

20. J. M. Weisberg, D. J. Nice and J. H. Taylor, ApJ $\mathbf{7 2 2}$ (October 2010) 1030, arXiv:1011.0718 [astro-ph.GA]

21. B. P. Abbott, R. Abbott, T. D. Abbott, M. R. Abernathy, F. Acernese, K. Ackley, C. Adams, T. Adams, P. Addesso, R. X. Adhikari and et al., Physical Review Letters 116 (February 2016) 061102, arXiv:1602.03837 [gr-qc].

22. B. P. Abbott, R. Abbott, T. D. Abbott, F. Acernese, K. Ackley, C. Adams, T. Adams, P. Addesso, R. X. Adhikari, V. B. Adya and et al., Physical Review Letters 119 (October 2017) 161101, arXiv:1710.05832 [gr-qc].

23. G.-B. Zhao, M. Raveri, L. Pogosian, Y. Wang, R. G. Crittenden, W. J. Handley, W. J. Percival, F. Beutler, J. Brinkmann, C.-H. Chuang, A. J. Cuesta, D. J. Eisenstein, F.-S. Kitaura, K. Koyama, B. L'Huillier, R. C. Nichol, M. M. Pieri, S. RodriguezTorres, A. J. Ross, G. Rossi, A. G. Sánchez, A. Shafieloo, J. L. Tinker, R. Tojeiro, J. A. Vazquez and H. Zhang, Nature Astronomy 1 (September 2017) 627, arXiv: 1701.08165 .

24. B. Ratra and P. J. E. Peebles, Phys. Rev. D 37 (June 1988) 3406.

25. E. J. Copeland, M. Sami and S. Tsujikawa, International Journal of Modern Physics D 15 (2006) 1753, hep-th/0603057.

26. A. Joyce, L. Lombriser and F. Schmidt, Annual Review of Nuclear and Particle Science 66 (October 2016) 95, arXiv:1601.06133.

27. R. Maartens and K. Koyama, Living Reviews in Relativity 13 (September 2010) 5, arXiv:1004.3962 [hep-th]

28. C. P. Burgess, ArXiv e-prints (September 2013) arXiv:1309.4133 [hep-th].

29. A. Padilla, ArXiv e-prints (February 2015) arXiv:1502.05296 [hep-th]. 
30. G. Dvali, G. Gabadadze and M. Porrati, Physics Letters B 485 (July 2000) 208, hep-th/0005016

31. M. A. Luty, M. Porrati and R. Rattazzi, Journal of High Energy Physics 9 (September 2003) 029, hep-th/0303116

32. D. Gorbunov, K. Koyama and S. Sibiryakov, Phys. Rev. D 73 (February 2006) 044016, hep-th/0512097.

33. C. M. Will, Living Reviews in Relativity 17 (June 2014) 4, arXiv:1403.7377 [gr-qc].

34. S. M. Merkowitz, Living Rev. Rel. 13 (2010) 7.

35. R. A. Hulse and J. H. Taylor, ApJL 195 (January 1975) L51.

36. G. D. Moore and A. E. Nelson, Journal of High Energy Physics 9 (September 2001) 023, hep-ph/0106220

37. L. Lombriser and A. Taylor, jcap 3 (March 2016) 031, arXiv:1509.08458

38. L. Lombriser and N. A. Lima, Physics Letters B 765 (February 2017) 382, arXiv:1602.07670.

39. D. Bettoni, J. M. Ezquiaga, K. Hinterbichler and M. Zumalacárregui, Phys. Rev. D 95 (April 2017) 084029, arXiv:1608.01982 [gr-qc].

40. P. Creminelli and F. Vernizzi, Physical Review Letters 119 (December 2017) 251302, arXiv:1710.05877.

41. J. Sakstein and B. Jain, Physical Review Letters 119 (December 2017) 251303, arXiv: 1710.05893 .

42. J. María Ezquiaga and M. Zumalacárregui, ArXiv e-prints (October 2017) arXiv:1710.05901.

43. T. Baker, E. Bellini, P. G. Ferreira, M. Lagos, J. Noller and I. Sawicki, Physical Review Letters 119 (December 2017) 251301, arXiv:1710.06394.

44. F. Sbisà, European Journal of Physics 36 (January 2015) 015009, arXiv:1406.4550 [hep-th]

45. A. Adams, N. Arkani-Hamed, S. Dubovsky, A. Nicolis and R. Rattazzi, Journal of High Energy Physics 10 (October 2006) 014, hep-th/0602178

46. C. de Rham, S. Melville, A. J. Tolley and S.-Y. Zhou, ArXiv e-prints (June 2017) arXiv:1706.02712 [hep-th].

47. R. Woodard, Avoiding Dark Energy with 1/R Modifications of Gravity, in The Invisible Universe: Dark Matter and Dark Energy, ed. L. Papantonopoulos, Lecture Notes in Physics, Berlin Springer Verlag, Vol. 720 (2007), p. 403. astro-ph/0601672.

48. D. Langlois and K. Noui, jcap 2 (February 2016) 034, arXiv:1510.06930 [gr-qc]

49. C. Wetterich, General Relativity and Gravitation 30 (January 1998) 159, gr-qc/9704052

50. S. Deser and R. P. Woodard, Physical Review Letters 99 (September 2007) 111301, arXiv:0706.2151.

51. M. Maggiore and M. Mancarella, Phys. Rev. D 90 (July 2014) 023005, arXiv: 1402.0448 [hep-th]

52. E. Belgacem, Y. Dirian, S. Foffa and M. Maggiore, ArXiv e-prints (December 2017) arXiv:1712.07066 [hep-th].

53. A. Emir Gümrükçüoğlu, M. Saravani and T. P. Sotiriou, Phys. Rev. D 97 (January 2018) 024032, arXiv:1711.08845 [gr-qc].

54. J. Oost, S. Mukohyama and A. Wang, ArXiv e-prints (February 2018) arXiv: 1802.04303 [gr-qc].

55. C. de Rham, Living Reviews in Relativity 17 (August 2014) 7, arXiv:1401.4173 [hep-th]

56. J. Khoury and A. Weltman, Physical Review Letters 93 (October 2004) 171104, 
astro-ph/0309300

57. J. Khoury and A. Weltman, Phys. Rev. D 69 (February 2004) 044026, astro-ph/0309411.

58. P. Brax, C. van de Bruck, A.-C. Davis and D. Shaw, Phys. Rev. D 82 (September 2010) 063519, arXiv:1005.3735

59. K. Hinterbichler and J. Khoury, Physical Review Letters 104 (June 2010) 231301, arXiv:1001.4525 [hep-th]

60. E. Babichev, C. Deffayet and R. Ziour, International Journal of Modern Physics D 18 (2009) 2147, arXiv:0905.2943 [hep-th]

61. A. I. Vainshtein, Physics Letters B 39 (May 1972) 393.

62. P. Brax, A.-C. Davis, B. Li and H. A. Winther, Phys. Rev. D 86 (August 2012) 044015, arXiv:1203.4812 [astro-ph.C0]

63. C. Burrage and J. Sakstein, ArXiv e-prints (September 2017) arXiv:1709.09071.

64. F. Rondeau and B. Li, Phys. Rev. D 96 (December 2017) 124009, arXiv:1709.07087 [gr-qc].

65. L. Amendola, Phys. Rev. D 62 (August 2000) 043511, astro-ph/9908023

66. C. Llinares and L. Pogosian, Phys. Rev. D 90 (December 2014) 124041, arXiv: 1410.2857

67. G.-B. Zhao, B. Li and K. Koyama, Physical Review Letters 107 (August 2011) 071303, arXiv:1105.0922 [astro-ph.CO].

68. A. Cabré, V. Vikram, G.-B. Zhao, B. Jain and K. Koyama, jcap 7 (July 2012) 034, arXiv:1204.6046

69. H. Desmond, P. G. Ferreira, G. Lavaux and J. Jasche, ArXiv e-prints (February 2018) arXiv:1802.07206.

70. P. Brax, A.-C. Davis and B. Li, Physics Letters B 715 (August 2012) 38, arXiv:1111.6613

71. W. Hu and I. Sawicki, Phys. Rev. D 76 (September 2007) 064004, arXiv:0705.1158

72. A. Barreira, P. Brax, S. Clesse, B. Li and P. Valageas, Phys. Rev. D 91 (June 2015) 123522, arXiv: 1504.01493.

73. V. I. Zakharov, Soviet Journal of Experimental and Theoretical Physics Letters 12 (1970) 312.

74. H. van Dam and M. J. G. Veltman, Nucl. Phys. B22 (1970) 397.

75. D. G. Boulware and S. Deser, Phys. Rev. D 6 (December 1972) 3368.

76. C. de Rham, G. Gabadadze and A. J. Tolley, Physical Review Letters 106 (June 2011) 231101, arXiv:1011.1232 [hep-th]

77. B. Li, G.-B. Zhao and K. Koyama, jcap 5 (May 2013) 023, arXiv:1303.0008 [astro-ph.CO].

78. A. Lue, R. Scoccimarro and G. D. Starkman, Phys. Rev. D 69 (June 2004) 124015, astro-ph/0401515

79. G. Dvali, A. Gruzinov and M. Zaldarriaga, Phys. Rev. D 68 (July 2003) 024012, hep-ph/0212069

80. T. Hiramatsu, W. Hu, K. Koyama and F. Schmidt, Phys. Rev. D 87 (March 2013) 063525, arXiv:1209.3364 [hep-th]

81. P. Brax, C. Burrage and A.-C. Davis, jcap 9 (September 2011) 020, arXiv:1106.1573 [hep-ph]

82. A. A. Starobinsky, Physics Letters B 91 (March 1980) 99

83. S. Nojiri and S. D. Odintsov, ArXiv e-prints (July 2008) arXiv:0807.0685 [hep-th]

84. T. P. Sotiriou and V. Faraoni, Reviews of Modern Physics 82 (January 2010) 451, arXiv:0805.1726 [gr-qc]

85. A. De Felice and S. Tsujikawa, Living Reviews in Relativity 13 (June 2010) 3, 
arXiv: 1002.4928 [gr-qc]

86. P. Brax, C. van de Bruck, A.-C. Davis and D. J. Shaw, Phys. Rev. D 78 (November 2008) 104021, arXiv:0806.3415

87. A. A. Starobinsky, Soviet Journal of Experimental and Theoretical Physics Letters 86 (October 2007) 157, arXiv:0706.2041.

88. S. Appleby and R. Battye, Physics Letters B 654 (October 2007) 7, arXiv:0705.3199.

89. L. Pogosian and A. Silvestri, Phys. Rev. D 77 (January 2008) 023503, arXiv:0709.0296.

90. J. Wang, L. Hui and J. Khoury, Physical Review Letters 109 (December 2012) 241301, arXiv: 1208.4612 [astro-ph.C0].

91. J. J. Ceron-Hurtado, J.-h. He and B. Li, Phys. Rev. D 94 (September 2016) 064052, arXiv:1609.00532,

92. Y.-S. Song, W. Hu and I. Sawicki, Phys. Rev. D 75 (February 2007) 044004, astro-ph/0610532.

93. C. Deffayet, G. Dvali and G. Gabadadze, Phys. Rev. D 65 (February 2002) 044023, astro-ph/0105068.

94. A. Lue and G. Starkman, Phys. Rev. D 67 (March 2003) 064002, astro-ph/0212083

95. K. Koyama and F. P. Silva, Phys. Rev. D 75 (April 2007) 084040, hep-th/0702169

96. A. Nicolis and R. Rattazzi, Journal of High Energy Physics 6 (June 2004) 059, hep-th/0404159

97. K. Koyama, Phys. Rev. D 72 (December 2005) 123511, hep-th/0503191

98. C. Charmousis, R. Gregory, N. Kaloper and A. Padilla, Journal of High Energy Physics 10 (October 2006) 066, hep-th/0604086.

99. C. Deffayet, G. Dvali, G. Gabadadze and A. Vainshtein, Phys. Rev. D 65 (February 2002) 044026, hep-th/0106001.

100. F. Schmidt, Phys. Rev. D 80 (August 2009) 043001, arXiv:0905.0858 [astro-ph.CO]

101. H. Ishihara, Physical Review Letters 86 (January 2001) 381, gr-qc/0007070.

102. G. W. Horndeski, Int. J. Theor. Phys. 10 (1974) 363.

103. A. Nicolis, R. Rattazzi and E. Trincherini, Phys. Rev. D 79 (March 2009) 064036, arXiv:0811.2197 [hep-th]

104. C. Deffayet, G. Esposito-Farèse and A. Vikman, Phys. Rev. D 79 (April 2009) 084003, arXiv:0901.1314 [hep-th]

105. C. Deffayet, X. Gao, D. A. Steer and G. Zahariade, Phys. Rev. D 84 (September 2011) 064039, arXiv:1103.3260 [hep-th]

106. C. Deffayet, S. Deser and G. Esposito-Farèse, Phys. Rev. D 80 (September 2009) 064015, arXiv:0906.1967 [gr-qc].

107. T. Kobayashi, M. Yamaguchi and J. Yokoyama, Physical Review Letters 105 (December 2010) 231302, arXiv:1008.0603 [hep-th]

108. C. Charmousis, E. J. Copeland, A. Padilla and P. M. Saffin, Physical Review Letters 108 (February 2012) 051101, arXiv:1106.2000 [hep-th].

109. C. Deffayet and D. A. Steer, Classical and Quantum Gravity 30 (November 2013) 214006, arXiv:1307.2450 [hep-th]

110. E. Bellini and I. Sawicki, jcap 7 (July 2014) 050, arXiv:1404.3713

111. C. Deffayet, O. Pujolàs, I. Sawicki and A. Vikman, jcap 10 (October 2010) 026, arXiv:1008.0048 [hep-th]

112. R. Kimura and K. Yamamoto, jcap 4 (April 2011) 025, arXiv:1011.2006.

113. R. Kimura, T. Kobayashi and K. Yamamoto, Phys. Rev. D 85 (June 2012) 123503, arXiv:1110.3598 [astro-ph.CO]. 
114. Z. Zhai, M. Blanton, A. Slosar and J. Tinker, ApJ 850 (December 2017) 183, arXiv:1705.10031.

115. H. Motohashi, K. Noui, T. Suyama, M. Yamaguchi and D. Langlois, jcap 7 (July 2016) 033, arXiv:1603.09355 [hep-th].

116. R. Klein and D. Roest, Journal of High Energy Physics 7 (July 2016) 130, arXiv:1604.01719 [hep-th].

117. M. Crisostomi, R. Klein and D. Roest, Journal of High Energy Physics 6 (June 2017) 124, arXiv: 1703.01623 [hep-th].

118. H. Motohashi, T. Suyama and M. Yamaguchi, ArXiv e-prints (November 2017) arXiv:1711.08125 [hep-th].

119. M. Zumalacárregui and J. García-Bellido, Phys. Rev. D 89 (March 2014) 064046, arXiv:1308.4685 [gr-qc]

120. J. Gleyzes, D. Langlois, F. Piazza and F. Vernizzi, Physical Review Letters 114 (May 2015) 211101, arXiv:1404.6495 [hep-th]

121. J. Gleyzes, D. Langlois, F. Piazza and F. Vernizzi, jcap 2 (February 2015) 018, arXiv:1408.1952,

122. D. Langlois and K. Noui, jcap 7 (July 2016) 016, arXiv:1512.06820 [gr-qc].

123. M. Crisostomi, K. Koyama and G. Tasinato, jcap 4 (April 2016) 044, arXiv:1602.03119 [hep-th].

124. J. Ben Achour, D. Langlois and K. Noui, Phys. Rev. D 93 (June 2016) 124005, arXiv: 1602.08398 [gr-qc]

125. J. Ben Achour, M. Crisostomi, K. Koyama, D. Langlois, K. Noui and G. Tasinato, ArXiv e-prints (August 2016) arXiv:1608.08135 [hep-th].

126. C. de Rham and A. Matas, jcap 6 (June 2016) 041, arXiv:1604.08638 [hep-th].

127. T. Kobayashi, Y. Watanabe and D. Yamauchi, Phys. Rev. D 91 (March 2015) 064013, arXiv: 1411.4130 [gr-qc].

128. D. Langlois, R. Saito, D. Yamauchi and K. Noui, ArXiv e-prints (November 2017) arXiv:1711.07403 [gr-qc]

129. M. Crisostomi and K. Koyama, Phys. Rev. D 97 (January 2018) 021301, arXiv:1711.06661.

130. K. Koyama and J. Sakstein, Phys. Rev. D 91 (June 2015) 124066, arXiv:1502.06872.

131. J. Beltrán Jiménez, F. Piazza and H. Velten, Physical Review Letters 116 (February 2016) 061101, arXiv:1507.05047 [gr-qc]

132. A. Dima and F. Vernizzi, ArXiv e-prints (December 2017) arXiv:1712.04731 [gr-qc].

133. R. Saito, D. Yamauchi, S. Mizuno, J. Gleyzes and D. Langlois, jcap 6 (June 2015) 008, arXiv: 1503.01448 [gr-qc]

134. J. Sakstein, Physical Review Letters 115 (November 2015) 201101, arXiv: 1510.05964.

135. J. Sakstein, Phys. Rev. D 92 (December 2015) 124045, arXiv:1511.01685.

136. M. Crisostomi and K. Koyama, ArXiv e-prints (December 2017) arXiv:1712.06556. 